\title{
Zigzag Persistence
}

\author{
Gunnar Carlsson • Vin de Silva
}

Received: 28 February 2009 / Accepted: 23 September 2009 / Published online: 21 April 2010

(C) The Author(s) 2010. This article is published with open access at Springerlink.com

\begin{abstract}
We describe a new methodology for studying persistence of topological features across a family of spaces or point-cloud data sets, called zigzag persistence. Building on classical results about quiver representations, zigzag persistence generalises the highly successful theory of persistent homology and addresses several situations which are not covered by that theory. In this paper we develop theoretical and algorithmic foundations with a view towards applications in topological statistics.
\end{abstract}

Keywords Applied topology $\cdot$ Persistent topology $\cdot$ Quiver representations

Mathematics Subject Classification (2000) 68W30 - 55N99

\section{Introduction}

\subsection{Overview}

In this paper, we describe a new methodology for studying persistence of topological features across a family of spaces or point-cloud data sets. This theory of zigzag persistence generalises the successful and widely used theory of persistence and persistent homology. Moreover, zigzag persistence can handle several important situations

Communicated by Herbert Edelsbrunner.

G. Carlsson $(\bowtie)$

Department of Mathematics, Stanford University, Stanford, CA, USA

e-mail: gunnar@math.stanford.edu

V. de Silva

Department of Mathematics, Pomona College, Claremont, CA, USA

e-mail: vin.desilva@pomona.edu 
that are not currently addressed by standard persistence. The technical basis for these developments comes from quiver theory [8].

The zigzag persistence framework is activated whenever one constructs a zigzag diagram of topological spaces or vector spaces: a sequence of spaces $S_{1}, \ldots, S_{n}$ where each adjacent pair is connected by a map $S_{i} \rightarrow S_{i+1}$ or $S_{i} \leftarrow S_{i+1}$. The novelty of our approach is that the direction of each linking map is arbitrary, in contrast to the usual theory of persistence where all maps point in the same direction.

This paper has three principal objectives:

- To describe several scenarios in applied topology where it is natural to consider zigzag diagrams (Sect. 1).

- To develop a mathematical theory of persistence for zigzag diagrams (Sects. 2 and 3 ).

- To develop algorithms for computing zigzag persistence (Sect. 4).

There is one subsidiary objective:

- To introduce the Diamond Principle, a calculational tool analogous in power and effect to the Mayer-Vietoris theorem in classical algebraic topology (Sect. 5).

Our goal is to present the fundamental concepts and constructions of zigzag persistence in an explicit and accessible way, and to provide a sound theoretical platform for future work. In particular, we draw the reader's attention to our paper with Morozov [3], where we develop a zigzag theory for topological spaces with a Morse-like function. This 'levelset zigzag persistence' can be related to the extended persistence of Cohen-Steiner et al. [6], and is proved to be stable under perturbations of the function.

We offer a few comments on what this paper does not do. On the computational side, we do not implement any algorithms or conduct a performance analysis. We make no attempt to provide an algorithm to compute zigzag persistence at the homological level; this is carried out in [3]. On the mathematical side, we do not claim new theoretical results in the underlying quiver representation theory. Rather, we use results implicit in the existing literature to provide a precise algorithmic framework for future code development projects. Finally, on a technical level, we are deeply indebted to the practitioners of quiver theory [8]; what is new here is the emphasis on algorithmics and on applications to computational topology (particularly Sects. 1, 4 and 5).

\subsection{Persistence}

One of the principal difficulties when attempting to apply algebraic topology to statistical data is the fact that traditional invariants - such as the Betti numbers or the fundamental group - are completely non-robust to discontinuous changes in the space under consideration. Indeed, they must be, to have discriminatory power. The challenge in topological statistics is to construct invariants which enjoy the discriminatory power of the classical invariants, but which have the necessary robustness for statistical use.

Persistent homology $[10,14]$ is the single most powerful existing tool for addressing this problem. 
A typical workflow runs as follows [7]. The input is a point cloud, that is, a finite subset of some Euclidean space or more generally a finite metric space. After an initial filtering step (to remove undesirable points or to focus on high-density regions of the data, say), a set of vertices is selected from the data, and a simplicial complex $S$ is built on that vertex set, according to some prearranged rule. In practice, the simplicial complex depends on a coarseness parameter $\epsilon$, and what we have is a nested family $\left\{S_{\epsilon}\right\}_{\epsilon \in[0, \infty]}$, which typically ranges from a discrete set of vertices at $S_{0}$ to a complete simplex at $S_{\infty}$.

Persistent homology takes the entire nested family $\left\{S_{\epsilon}\right\}$ and produces a barcode or persistence diagram as output. A barcode is a collection of half-open subintervals $\left[b_{j}, d_{j}\right) \subseteq[0, \infty)$, which describes the homology of the family as it varies over $\epsilon$. An interval $\left[b_{j}, d_{j}\right)$ represents a homological feature which is born at time $b_{j}$ and dies at time $d_{j}$. This construction has several excellent properties:

- There is no need to select a particular value of $\epsilon$.

- Features can be evaluated by interval length. Long intervals are regarded as indicating essential features of the data, whereas short intervals are likely to be artefacts of noise.

- There exists a fast algorithm to compute the barcode [10, 14].

- The barcode is a complete invariant of the homology of the family of complexes [14].

- The barcode is provably stable with respect to changes in the input $[4,5]$. In contrast, any individual homology group $\mathrm{H}_{k}\left(S_{\epsilon}\right)$ is highly unstable.

The major limitation of persistence is that it depends crucially on the family $\left\{S_{\epsilon}\right\}$ being nested, in the sense that $S_{\epsilon} \subseteq S_{\epsilon^{\prime}}$ whenever $\epsilon \leq \epsilon^{\prime}$. This applies to the current theoretical understanding as well as the algorithms. Zigzag persistence addresses this limitation.

If we discretise the variable $\epsilon$ to a finite set of values, the family of simplicial complexes can be thought of as a diagram of spaces

$$
S_{1} \rightarrow S_{2} \rightarrow \cdots \rightarrow S_{n}
$$

where the arrows represent the inclusion maps. If we apply the $k$-dimensional homology functor $\mathrm{H}_{k}(-; \mathrm{k})$ with coefficients in a field $\mathrm{k}$, this becomes a diagram of vector spaces

$$
V_{1} \rightarrow V_{2} \rightarrow \cdots \rightarrow V_{n}
$$

and linear maps, where $V_{i}=\mathrm{H}_{k}\left(S_{i} ; \mathrm{k}\right)$. Such a diagram is called a persistence module. What makes persistence work is that there is a simple algebraic classification of persistence modules up to isomorphism; each possible barcode corresponds to an isomorphism type.

Our goal is to achieve a similar classification for diagrams in which the arrows may point in either direction. This is zigzag persistence, in a nutshell.

\subsection{Zigzag Diagrams in Applied Topology}

We consider some problems which arise quite naturally in the computational topology of data. 
Example 1.1 Some of the most interesting properties of a point cloud are contained in the estimates of the probability density from which the data are sampled. Deep structure is sometimes revealed after thresholding according to a density estimate (see [2] for an example drawn from visual image analysis). However, the construction of a density estimation function $\rho$ invariably depends on choosing a smoothing parameter: for instance $\rho(x)$ might be defined to be the number of data points within distance $r$ of $x$; here $r$ is the smoothing parameter.

It happens that different choices of smoothing parameter may well reveal different structures in the data; a particularly striking example of this occurs in [2]. Statisticians have invented useful criteria for determining what the 'appropriate' value of such a parameter might be for a particular data set; but another point of view would be to analyse all values of the parameter simultaneously, and to study how the topology changes as the parameter varies.

The problem with doing this is that there is no natural relationship between, say, the $25 \%$ densest points as measured using two different parameter values. This means that one cannot build an increasing family of spaces using the change in parameters, and so one cannot use persistence to analyse the evolution of the topology. On the other hand, there are natural zigzag sequences which can be used to study this problem. Select a sequence of parameter values $r_{1}<r_{2}<\cdots<r_{n}$ and a percentage $p$, and let $X_{r}^{p}$ denote the densest $p \%$ of the point cloud when measured according to parameter value $r$. We can then consider the union sequence

$$
X_{r_{1}}^{p} \rightarrow X_{r_{1}}^{p} \cup X_{r_{2}}^{p} \leftarrow X_{r_{2}}^{p} \rightarrow X_{r_{2}}^{p} \cup X_{r_{3}}^{p} \leftarrow X_{r_{3}}^{p} \rightarrow \cdots \leftarrow X_{r_{n}}^{p}
$$

or the intersection sequence

$$
X_{r_{1}}^{p} \leftarrow X_{r_{1}}^{p} \cap X_{r_{2}}^{p} \rightarrow X_{r_{2}}^{p} \leftarrow X_{r_{2}}^{p} \cap X_{r_{3}}^{p} \rightarrow X_{r_{3}}^{p} \leftarrow \cdots \rightarrow X_{r_{n}}^{p}
$$

As we shall see in Sect. 5.3, there is essentially no difference between the zigzag persistent homology of the union and intersection sequences of a sequence of spaces. Here that assertion needs to be filtered through the process of representing the data subsets $X_{r}^{p}$ as simplicial complexes.

Example 1.2 (Topological bootstrapping) Suppose we are given a very large point cloud $X$. If it is too large to process directly, we may take a sequence of small samples $X_{1}, \ldots, X_{n}$ and estimate their topology individually, perhaps obtaining a persistence barcode for each one. How does this reflect the topology of the original sample $X$ ? On one hand, if most of the barcodes have similar appearance, then one might suppose that $X$ itself will have the same barcode. On the other hand, one needs to be able to distinguish between a single feature detected repeatedly, and multiple features detected randomly but one at a time. If we detect $n$ features in $X_{i}$ on average, are we detecting $n$ features of $X$ with detection probability 1 , or $k n$ features with detection probability $1 / k$ ?

Once again, there is a need to correlate features across different instances of the construction. The union sequence comes to the rescue:

$$
X_{1} \rightarrow X_{1} \cup X_{2} \leftarrow X_{2} \rightarrow \cdots \leftarrow X_{n}
$$


In this case, the intersection sequence is not useful at the level of samples, because two sparse samples are unlikely to intersect very much.

The approach in this example is analogous to bootstrapping in statistics, where measurements on a large data set are estimated by making repeated measurements on a set of samples.

Example 1.3 In computational topology, there exist several techniques for modelling a point-cloud data set $X$ by a simplicial complex $S$ : the Cech complex, the VietorisRips complex, the alpha complex [9], the witness complex [7], and so on. The witness complex $W(L ; X)$, in particular, depends on the choice of a small subset of 'landmark' points $L \subset X$ which will serve as the vertex set of $S$. Roughly speaking, a simplex $\sigma$ with vertices in $L$ is included in $W(L ; X)$ if there is some $x \in X$ which witnesses it, by being close to all the vertices.

How does the witness complex $W(L ; X)$ depend on the choice of landmark set? There is no direct way to compare $W(L ; X)$ with $W(M ; X)$ for two different choices of landmark sets $L, M$. However, it turns out that one can define a witness bicomplex $W(L, M ; X)$ which maps onto each witness complex. The cells are Cartesian products $\sigma \times \tau$, where $\sigma, \tau$ have vertices in $L, M$ respectively. A cell $\sigma \times \tau$ is included provided that there exists $x \in X$ which simultaneously witnesses $\sigma$ for $W(L ; X)$ and $\tau$ for $W(M ; X)$.

Given a sequence $L_{1}, \ldots, L_{n}$ of landmark subsets, one can then construct the witness bicomplex zigzag:

$$
W\left(L_{1} ; X\right) \leftarrow W\left(L_{1}, L_{2} ; X\right) \rightarrow W\left(L_{2} ; X\right) \leftarrow \cdots \rightarrow W\left(L_{n} ; X\right) .
$$

Long intervals in the zigzag barcode will then indicate features that persist across the corresponding range of choices of landmark set.

The fundamental requirement is then for a way of assessing, in a zigzag diagram of vector spaces, the degree to which consistent families of elements exist. The point of this paper is that there is such methodology. We will interpret the isomorphism classes of zigzag diagrams as a special case of the classification problem for quiver representations (see [8] for background on this theory). There turns out to be a theorem of Gabriel [11] which classifies arbitrary diagrams based on Dynkin diagrams, and which shows in particular that the set of isomorphism classes of zigzag diagrams of a given length is parametrised by barcodes-just as persistence modules are. Long intervals in the classification define large families of consistent elements, hence indicate the presence of features stable across samples, landmark sets, or parameter values for a density estimator.

\subsection{Organisation of the Paper}

In Sect. 2 we describe the theory of decompositions of zigzag modules. These decompositions produce zigzag persistence barcodes analogous to the barcodes of persistent homology. The foundational theorem of Gabriel is stated without proof. In Sect. 3 we develop the machinery of right-filtrations, which turn out to be the right tool for accessing the decomposition structure of a zigzag module. This is an important section 
for the reader who wishes to make serious use of zigzag persistence. In Sect. 4, we present a general-purpose algorithmic framework for calculating zigzag persistence, and we show how this operates in a practical class of examples. The algorithm is based on a proof of Gabriel's theorem for zigzag modules, included for completeness. Section 5 is devoted to a localisation principle which gives another approach to zigzag barcode calculations. We apply this to prove the Diamond Principle. We use this in turn to compare the zigzag barcodes for two natural zigzag diagrams obtained from a sequence of simplicial complexes.

\section{Zigzag Diagrams of Vector Spaces}

We work over a field $k$ which remains fixed throughout this paper. There is no significance to the choice of $\mathrm{k}$. All vector spaces are finite-dimensional.

\subsection{Zigzag Modules}

Let $\mathbb{V}$ denote a sequence of vector spaces and linear maps, of length $n$ :

$$
V_{1} \stackrel{p_{1}}{\longleftrightarrow} V_{2} \stackrel{p_{2}}{\longleftrightarrow} \cdots \stackrel{p_{n-1}}{\longleftrightarrow} V_{n} .
$$

Each $\stackrel{p_{i}}{\longleftrightarrow}$ represents either a forward map $\stackrel{f_{i}}{\longrightarrow}$ or a backward map $\stackrel{g_{i}}{\longleftarrow}$. The object $\mathbb{V}$ is called a zigzag diagram of vector spaces, or simply a zigzag module, over $\mathrm{k}$.

The sequence of symbols $f$ or $g$ is the type of $\mathbb{V}$. For instance, a diagram of type $\tau=f g g$ looks like this:

$$
V_{1} \stackrel{f_{1}}{\longrightarrow} V_{2} \stackrel{g_{2}}{\longleftarrow} V_{3} \stackrel{g_{3}}{\longleftarrow} V_{4} .
$$

The length of a type $\tau$ is the length of any diagram of type $\tau$. For example, we say that $f g g$ has length 4 . We will usually be considering zigzag modules of a fixed type $\tau$ of length $n$. Such diagrams are called $\tau$-modules, and the class of $\tau$-modules is denoted $\tau$ Mod.

Persistence modules (see [10,14]) are zigzag modules where all the maps have the forward orientation; in other words, where $\tau=f f \ldots f$. As explained in [14], persistence modules can be viewed as graded modules over the polynomial ring $\mathrm{k}[t]$. This observation simplifies the analysis of persistence modules quite considerably.

More generally, one can consider graph representations of arbitrary oriented graphs. Zigzag modules constitute the special case where the graph is $A_{n}$ (a path with $n$ vertices and $n-1$ edges) and the orientation is specified by the type $\tau$. In 1972 , Gabriel showed that the Dynkin-Coxeter graphs $A_{n}, D_{n}, E_{6}, E_{7}, E_{8}$ (arbitrarily oriented) have an especially well-behaved representation theory [11]. The theory of quivers was launched from this starting block; see [8] for a beautiful and transparent introduction. Zigzag persistence is enabled by the good behaviour of $A_{n}$ graph representations.

Remark $\tau$ Mod has the structure of an abelian category. Given two $\tau$-modules $\mathbb{V}, \mathbb{W}$, a morphism $\alpha: \mathbb{V} \rightarrow \mathbb{W}$ is defined to be a collection of linear maps $\alpha_{i}: V_{i} \rightarrow W_{i}$ 
which satisfy the commutation relations $\alpha_{i+1} f_{i}=h_{i} \alpha_{i}$ or $\alpha_{i} g_{i}=k_{i} \alpha_{i+1}$ for each $i$. (Here the forward and backward maps for $\mathbb{W}$ are written $h, k$ respectively.) Morphisms can be composed in the obvious way, and have kernels, images, and cokernels: for instance $\mathbb{K}=\operatorname{Ker}(\alpha)$ is the $\tau$-module with spaces $K_{i}=\operatorname{Ker}\left(V_{i} \rightarrow W_{i}\right)$ and maps $\left.f_{i}\right|_{K_{i}}$ and $\left.g_{i}\right|_{K_{i+1}}$ defined by restriction. The set of morphisms $\operatorname{Hom}(\mathbb{V}, \mathbb{W})$ is naturally a vector space over $\mathrm{k}$, and the endomorphism $\operatorname{ring} \operatorname{End}(\mathbb{V})=\operatorname{Hom}(\mathbb{V}, \mathbb{V})$ is a non-commutative k-algebra. We can view $\operatorname{End}(\mathbb{V})$ as the subalgebra of $\operatorname{End}\left(V_{1}\right) \times$ $\cdots \times \operatorname{End}\left(V_{n}\right)$ defined by the commutation relations.

\subsection{Decompositions of Zigzag Modules}

We wish to understand zigzag modules by decomposing them into simpler parts. Accordingly, a submodule $\mathbb{W}$ of a $\tau$-module $\mathbb{V}$ is defined by subspaces $W_{i} \leq V_{i}$ such that $f_{i}\left(W_{i}\right) \leq W_{i+1}$ or $g_{i}\left(W_{i+1}\right) \leq W_{i}$ for all $i$. These conditions guarantee that $\mathbb{W}$ is itself a $\tau$-module, with maps given by the restrictions $\left.f_{i}\right|_{W_{i}}$ or $\left.g_{i}\right|_{W_{i+1}}$. We write $\mathbb{W} \leq \mathbb{V}$.

A submodule $\mathbb{W}$ is called a summand of $\mathbb{V}$ if there exists a submodule $\mathbb{X} \leq \mathbb{V}$ which is complementary to $\mathbb{W}$, in the sense that $V_{i}=W_{i} \oplus X_{i}$ for all $i$. In that case, we say that $\mathbb{V}$ is the direct sum of $\mathbb{W}, \mathbb{X}$ and write $\mathbb{V}=\mathbb{W} \oplus \mathbb{X}$.

Example 2.1 As a rule, most submodules are not summands. $\mathbb{V}=(\mathrm{k} \stackrel{1}{\longrightarrow} \mathrm{k})$ has the submodule $\mathbb{W}=(0 \longrightarrow k)$. However, $\mathbb{W}$ is not a summand because the only possible complement is $(\mathrm{k} \longrightarrow 0)$, and that is not a submodule of $\mathbb{V}$.

Remark The direct sum can also be defined as an 'external' operation: given $\tau$-modules $\mathbb{V}, \mathbb{W}$ their direct sum $\mathbb{V} \oplus \mathbb{W}$ is defined to be the $\tau$-module with spaces $V_{i} \oplus W_{i}$ and maps $f_{i} \oplus h_{i}$ or $g_{i} \oplus k_{i}$. (Here the forward and backward maps for $\mathbb{W}$ are written $h, k$ respectively.)

A $\tau$-module $\mathbb{V}$ is decomposable if it can be written as a direct sum of nonzero submodules, and indecomposable otherwise. Any $\tau$-module $\mathbb{V}$ has a Remak decomposition; in other words we can write $\mathbb{V}=\mathbb{W}_{1} \oplus \cdots \oplus \mathbb{W}_{N}$, where the summands $\mathbb{W}_{j}$ are indecomposable. The existence of such a decomposition is proved by induction on the total dimension $\sum_{i} \operatorname{dim}\left(V_{i}\right)$ : if $\mathbb{V}$ is decomposable, say $\mathbb{V}=\mathbb{W} \oplus \mathbb{X}$, then we may assume inductively that $\mathbb{W}, \mathbb{X}$ have Remak decompositions, and therefore so does $\mathbb{V}$. (Base case: if $\mathbb{V}$ is indecomposable, then it has a Remak decomposition with one term.)

Remak decompositions themselves are not unique. However, the Krull-Schmidt principle tells us that the summands in a Remak decomposition are unique up to reordering:

Proposition 2.2 (Krull-Remak-Schmidt) Suppose a $\tau$-module $\mathbb{V}$ has Remak decompositions

$$
\mathbb{V}=\mathbb{W}_{1} \oplus \cdots \oplus \mathbb{W}_{M} \quad \text { and } \quad \mathbb{V}=\mathbb{X}_{1} \oplus \cdots \oplus \mathbb{X}_{N}
$$

Then $M=N$ and there is some permutation $\sigma$ of $\{1, \ldots, N\}$ such that $\mathbb{W}_{j} \cong \mathbb{X}_{\sigma(j)}$ for all $j$. 
Proof The proof of Theorem 7.5 of Lang [13], which is stated for modules in the ordinary sense, can be applied verbatim to our present context; all the required algebraic operations can be carried out within $\operatorname{End}(\mathbb{V})$. Since our $\tau$-modules have finite total dimension, the ascending and descending chain conditions (ACC and DCC) are automatic.

For further context, we refer the reader to an elegant article by Atiyah [1]; the Krull-Schmidt principle applies in any exact abelian category to objects which satisfy ACC and DCC, or a weaker 'bi-chain condition' defined by Atiyah. Our category, $\tau$ Mod, is included by this formulation.

Thus we can use the multiset $\left\{\mathbb{W}_{j}\right\}$ as an isomorphism invariant of $\mathbb{V}$. For this to be useful, we need to identify the set of indecomposable $\tau$-modules. We now describe a natural collection of indecomposables. For each subinterval $[b, d]$ of the integer sequence $\{1, \ldots, n\}$ there is an associated $\tau$-module.

Definition 2.3 Let $\tau$ be a type of length $n$ and consider integers $1 \leq b \leq d \leq n$. The interval $\tau$-module with birth time $b$ and death time $d$ is written $\mathbb{I}_{\tau}(b, d)$ and defined with spaces

$$
I_{i}= \begin{cases}\mathrm{k} & \text { if } b \leq i \leq d, \\ 0 & \text { otherwise }\end{cases}
$$

and with identity maps between adjacent copies of $k$, and zero maps otherwise. When $\tau$ is implicit, we will usually suppress it and simply write $\mathbb{I}(b, d)$.

Example If $\tau=f g g$ then $\mathbb{I}(2,3)$ is the zigzag module

$$
0 \stackrel{0}{\longrightarrow} \mathrm{k} \stackrel{1}{\longleftarrow} \mathrm{k} \stackrel{0}{\longleftarrow} 0
$$

Proposition 2.4 Interval $\tau$-modules are indecomposable.

Proof Suppose $\mathbb{I}(b, d)=\mathbb{V} \oplus \mathbb{W}$ and consider two adjacent terms $\mathrm{k}$ connected by an identity map. Since $\mathbb{V}, \mathbb{W}$ are submodules, the dimensions of $\mathbb{V}$ and $\mathbb{W}$ cannot decrease in the direction of the map; nor, since they are complements, can they increase. Thus $\operatorname{dim}\left(V_{i}\right)$ and $\operatorname{dim}\left(W_{i}\right)$ are constant over $b \leq i \leq d$, and in particular one of $\mathbb{V}, \mathbb{W}$ must be zero.

Here is the foundation stone for the theory of zigzag persistence.

Theorem 2.5 (Gabriel) The indecomposable $\tau$-modules are precisely the intervals $\mathbb{I}(b, d)$, where $1 \leq b \leq d \leq n=$ length $(\tau)$. Equivalently, every $\tau$-module can be written as a direct sum of intervals.

Proof This is the simplest special case of Gabriel's theorem, for the graphs $A_{n}$. The original reference (in German) is [11]. See [8] for an accessible overview. 
Thus, any $\tau$-module can be described completely up to isomorphism as an unordered list of intervals $[b, d]$, which correspond to its indecomposable summands. This is in exact accordance with the special case of ordinary persistence, where the result is comparatively easy to prove: it is simply the classification of finitely-generated graded modules over the polynomial ring $\mathrm{k}[t]$ (see [14]).

The philosophical point is that the decomposition theory of graph representations is somewhat independent of the orientation of the graph edges (see Kac [12]). Even in our case this is surprising, because there is no obvious congruence between persistence modules and zigzag modules of an arbitrary type $\tau$. However, if we accept this principle, then the generalisation from ordinary persistence to zigzag persistence is not surprising: interval decomposition for persistence modules implies interval decomposition for zigzag modules.

We will devote much of this paper to constructing a stand-alone proof of Theorem 2.5. This provides technical support towards our two main goals: to provide algorithms for computing the interval summands of a given $\tau$-module; and to make rigorous statements about the output of those algorithms.

\subsection{Zigzag Persistence}

We now define zigzag persistence and develop some of its elementary properties.

Definition 2.6 Let $\mathbb{V}$ be a zigzag module (of arbitrary type). The zigzag persistence of $\mathbb{V}$ is defined to be the multiset

$$
\operatorname{Pers}(\mathbb{V})=\left\{\left[b_{j}, d_{j}\right] \subseteq\{1, \ldots, n\} \mid j=1, \ldots, N\right\}
$$

of integer intervals derived from a decomposition $\mathbb{V} \cong \mathbb{I}\left(b_{1}, d_{1}\right) \oplus \cdots \oplus \mathbb{I}\left(b_{N}, d_{N}\right)$. The Krull-Schmidt principle asserts that this definition is independent of the decomposition.

Graphically, Pers $(\mathbb{V})$ can be represented as a set of lines measured against a single axis with labels $\{1, \ldots, n\}$ (the barcode), or as a multiset of points in $\mathbb{R}^{2}$ lying on or above the diagonal in the positive quadrant (the persistence diagram). See Fig. 1 for an example presented in each style.

Remark In the special case of persistence modules, this agrees with the standard treatment (see $[10,14])$ except in the following particular: the closed integer intervals $\left[b_{j}, d_{j}\right] \subseteq\{1, \ldots, n\}$ are replaced by half-open real intervals $\left[b_{j}, d_{j}+1\right) \subset \mathbb{R}$

Fig. 1 Barcode (left) and persistence diagram (right) representations of the persistence $\{[1,2],[1,3],[3,3],[3,4],[3,4]\}$ of a zigzag module of length 4
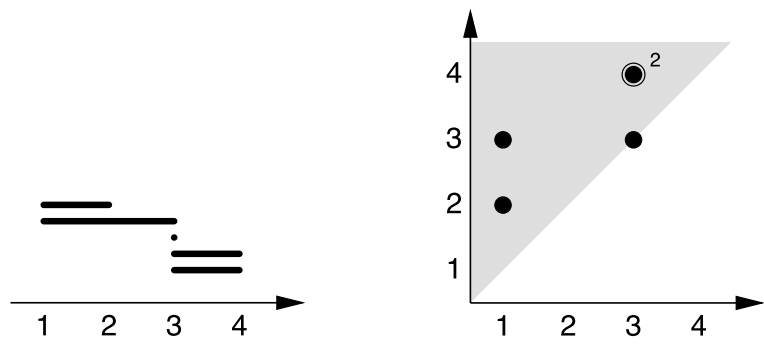
in the standard treatment. This is particularly natural when the indexing parameter is continuous: an interval $[b, d)$ indicates a feature born at time $b$ that survives right up to, but vanishes at, time $d$. Our convention is motivated by the desire to maintain symmetry between the forward and backward directions. We advise the reader to take particular care in handling the different conventions.

The transition from a zigzag module to its interval decomposition presents certain hazards which are not present in the case of persistence modules. We now draw attention to these hazards.

Definition 2.7 Let $\mathbb{V}$ be a zigzag module and let $\mathbb{V}[p, q]$ denote the restriction of $\mathbb{V}$ to the index set $p \leq i \leq q$. A feature of $\mathbb{V}$ over the time interval $[p, q]$ is a summand of $\mathbb{V}[p, q]$ isomorphic to $\mathbb{I}(p, q)$.

With persistence modules, there are several equivalent ways to recognise the existence of a feature. Here is a sample result.

Proposition 2.8 Let $\mathbb{V}$ be a persistence module of length $n$, and let $1 \leq p \leq q \leq n$. The following are equivalent:

(1) The composite map $V_{p} \rightarrow V_{q}$ is nonzero.

(2) There exist nonzero elements $x_{i} \in V_{i}$ for $p \leq i \leq q$, such that $x_{i+1}=f_{i}\left(x_{i}\right)$ for $p \leq i<q$.

(3) There exists a submodule of $\mathbb{V}[p, q]$ isomorphic to $\mathbb{I}(p, q)$.

(4) There exists a summand of $\mathbb{V}[p, q]$ isomorphic to $\mathbb{I}(p, q)$, i.e. a feature over $[p, q]$.

Proof It is easy to verify that (1), (2), (3) are equivalent. For (1) $\Rightarrow$ (2), begin by choosing $x_{p} \in V_{p}$ that maps to a nonzero element in $V_{q}$, and let $x_{i}$ be the image of $x_{p}$ in $V_{i}$. For $(2) \Rightarrow(3)$, define $\mathbb{I}$ by $I_{i}=\operatorname{Span}\left(x_{i}\right)$. For $(3) \Rightarrow(1)$, note that the restriction $I_{p} \rightarrow I_{q}$ is nonzero.

Clearly (4) $\Rightarrow(3)$. We now show that $(1) \Rightarrow(4)$. Consider an interval decomposition $\mathbb{V}[p, q]=\mathbb{I}\left(b_{1}, d_{1}\right) \oplus \cdots \oplus \mathbb{I}\left(b_{N}, d_{N}\right)$. On each summand, the map $I_{p}\left(b_{j}, d_{j}\right) \rightarrow I_{q}\left(b_{j}, d_{j}\right)$ is zero unless $b_{j}=p$ and $d_{j}=q$. Thus at least one of the summands is isomorphic to $\mathbb{I}(p, q)$.

The intuitions supported by Proposition 2.8 break down in the general case.

Caution 2.9 Let $\mathbb{V}$ be a zigzag module of arbitrary type. Statement (1) has no clear interpretation at this stage (something can be said in terms of the right-filtration functor of Sect. 3). Consider the following statements:

(2) There exist nonzero elements $x_{i} \in V_{i}$ for $p \leq i \leq q$, such that $x_{i+1}=f_{i}\left(x_{i}\right)$ or $x_{i}=g_{i}\left(x_{i+1}\right)$ (whichever is applicable) for $p \leq i<q$.

(3) There exists a submodule of $\mathbb{V}[p, q]$ isomorphic to $\mathbb{I}(p, q)$.

(4) There exists a summand of $\mathbb{V}[p, q]$ isomorphic to $\mathbb{I}(p, q)$, i.e. a feature over $[p, q]$. 
It is easy to verify that (2) $\Leftrightarrow(3)$ and that (4) implies (2), (3). However, the next two examples demonstrate that (2), (3) do not in general imply (4).

Example 2.10 Let $\tau=g f$ and consider the $\tau$-module $\mathbb{V}$ defined as follows:

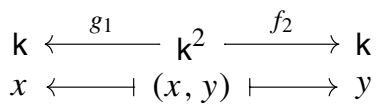

The interval decomposition is $\mathbb{V}=\mathbb{I}(1,2) \oplus \mathbb{I}(2,3)$, where the summands are

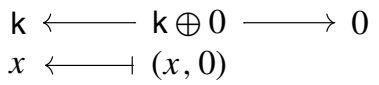

and

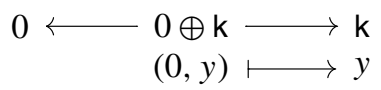

respectively. If this example appeared in a statistical topology setting, the feature corresponding to the generator of the $\mathrm{k}$ at $V_{1}$ would be regarded as unrelated to the feature corresponding to the generator of the $\mathrm{k}$ at $V_{3}$.

On the other hand, $\mathbb{V}$ does have a submodule (in fact, many submodules) isomorphic to $\mathbb{I}(1,3)$. Indeed, let $\Delta=\{(x, x) \mid x \in \mathrm{k}\}$ denote the diagonal subspace of $\mathrm{k}^{2}$. Then

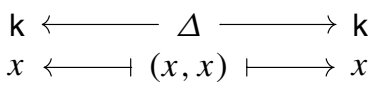

is a submodule $\mathbb{W} \leq \mathbb{V}$ isomorphic to $\mathbb{I}(1,3)$. The quotient $\tau$-module $\mathbb{V} / \mathbb{W}$ is isomorphic to $\mathbb{I}(2,2)$ but $\mathbb{W}$ has no complementary $\tau$-module in $\mathbb{V}$. Indeed, that would contradict the Krull-Schmidt theorem. More concretely, any complement of $\mathbb{W}$ must be isomorphic to $(0 \longleftarrow \mathrm{k} \longrightarrow 0)$, but that would require a 1-dimensional subspace of $\operatorname{Ker}\left(g_{1}\right) \cap \operatorname{Ker}\left(f_{2}\right)=0$.

Example 2.11 We can extend the previous example to arbitrary length. Consider the type $\tau=g f \ldots g f=(g f)^{n}$, of length $2 n+1$. Let $\mathbb{V}$ be the $\tau$-module

$$
\mathrm{k} \stackrel{\pi_{1}}{\longleftarrow} \mathrm{k}^{2} \stackrel{\pi_{2}}{\longrightarrow} \mathrm{k} \stackrel{\pi_{1}}{\longleftarrow} \cdots \stackrel{\pi_{2}}{\longrightarrow} \mathrm{k} \stackrel{\pi_{1}}{\longleftarrow} \mathrm{k}^{2} \stackrel{\pi_{2}}{\longrightarrow} \mathrm{k},
$$

where $\pi_{1}(x, y)=x$, and $\pi_{2}(x, y)=y$. Then $\mathbb{V}$ is isomorphic to a sum of short intervals

$$
\mathbb{I}(1,2) \oplus\{\mathbb{I}(2,4) \oplus \cdots \oplus \mathbb{I}(2 n-2,2 n)\} \oplus \mathbb{I}(2 n, 2 n+1)
$$

but it has a submodule

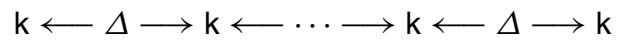

isomorphic to the long interval $\mathbb{I}(1,2 n+1)$. 
Moral In zigzag persistence it is necessary to respect the distinction between submodules and summands. Features are defined in terms of summands; never submodules.

We have defined features in terms of a chosen subinterval $[p, q]$. Features behave as expected when zooming to a larger or smaller window of observation. The following proposition illustrates what we mean.

Proposition 2.12 Let $\mathbb{V}$ be a zigzag module of length $n$ and let $1 \leq p \leq q \leq n$. The following statements are equivalent:

(1) There exists a summand of $\mathbb{V}[p, q]$ isomorphic to $\mathbb{I}(p, q)$, i.e. a feature over $[p, q]$.

(2) There exists a summand of $\mathbb{V}$ isomorphic to $\mathbb{I}\left(p^{\prime}, q^{\prime}\right)$, for some $\left[p^{\prime}, q^{\prime}\right] \supseteq[p, q]$.

Indeed, there is a bijection between intervals $[p, q]$ in $\operatorname{Pers}(\mathbb{V}[p, q])$ and intervals $\left[p^{\prime}, q^{\prime}\right] \supseteq[p, q]$ in $\operatorname{Pers}(\mathbb{V})$.

Proof Consider an interval decomposition $\mathbb{V}=\mathbb{I}\left(b_{1}, d_{1}\right) \oplus \cdots \oplus \mathbb{I}\left(b_{N}, d_{N}\right)$. By restriction, this induces an interval decomposition of $\mathbb{V}[p, q]$ into intervals $\mathbb{I}\left(b_{j}, d_{j}\right)[p, q]$. This induces the claimed bijection, because $\left[b_{j}, d_{j}\right]$ restricts to $[p, q]$ if and only if $\left[b_{j}, d_{j}\right] \supseteq[p, q]$.

Operating invisibly in this proof is the Krull-Schmidt principle, which allows us to select the interval decompositions most convenient to us when calculating Pers $(\mathbb{V})$ and Pers $(\mathbb{V}[p, q])$.

Remark 2.13 Sometimes it is useful to reduce the resolution of Pers( $\mathbb{V})$. Let $K \subset$ $\{1, \ldots, n\}$ be any subset. We define the restriction of Pers $(\mathbb{V})$ to $K$ to be the multiset

$$
\left.\operatorname{Pers}(\mathbb{V})\right|_{K}=\{I \cap K \mid I \in \operatorname{Pers}(\mathbb{V}), I \cap K \neq \emptyset\} .
$$

For instance, Proposition 2.12 amounts to the observation that $\operatorname{Pers}(\mathbb{V}[p, q])=$ $\left.\operatorname{Pers}(\mathbb{V})\right|_{[p, q]}$.

\section{From Zigzag Modules to Filtrations}

\subsection{The Right-Filtration Operator}

Our strategy is to understand (and construct) decompositions of a $\tau$-module $\mathbb{V}$ by an iterative process, moving from left to right and retaining the necessary information at each stage. The bulk of this information is encoded as a filtration on the rightmost vector space $V_{n}$.

Definition 3.1 The right-filtration $\mathrm{R}(\mathbb{V})$ of a $\tau$-module $\mathbb{V}$ of length $n$ takes the form

$$
\mathrm{R}(\mathbb{V})=\left(R_{0}, R_{1}, \ldots, R_{n}\right),
$$


where the $R_{i}$ are subspaces of $V_{n}$ satisfying the inclusion relations

$$
0=R_{0} \leq R_{1} \leq \cdots \leq R_{n}=V_{n}
$$

$\mathrm{R}(\mathbb{V})$ is defined recursively as follows.

Base case:

- If $\mathbb{V}$ has length 1 , then $\mathrm{R}(\mathbb{V})=\left(0, V_{1}\right)$.

Recursive step. Suppose we have already defined $\mathrm{R}(\mathbb{V})=\left(R_{0}, R_{1}, \ldots, R_{n}\right)$ :

- If $\mathbb{V}^{+}$is $\mathbb{V} \stackrel{f_{n}}{\longrightarrow} V_{n+1}$, then $\mathrm{R}\left(\mathbb{V}^{+}\right)=\left(f_{n}\left(R_{0}\right), f_{n}\left(R_{1}\right), \ldots, f_{n}\left(R_{n}\right), V_{n+1}\right)$.

- If $\mathbb{V}^{+}$is $\mathbb{V} \stackrel{g_{n}}{\longleftarrow} V_{n+1}$, then $\mathrm{R}\left(\mathbb{V}^{+}\right)=\left(0, g_{n}^{-1}\left(R_{0}\right), g_{n}^{-1}\left(R_{1}\right), \ldots, g_{n}^{-1}\left(R_{n}\right)\right)$.

To verify that $\mathrm{R}\left(\mathbb{V}^{+}\right)$is a filtration of the specified form, note that $R_{i} \leq R_{i+1}$ implies that $f_{n}\left(R_{i}\right) \leq f_{n}\left(R_{i+1}\right)$ in the first case, and $g_{n}^{-1}\left(R_{i}\right) \leq g_{n}^{-1}\left(R_{i+1}\right)$ in the second case. Moreover $f_{n}\left(R_{0}\right)=f_{n}(0)=0$, and $g_{n}^{-1}\left(R_{n}\right)=g_{n}^{-1}\left(V_{n}\right)=V_{n+1}$.

Example 3.2 Here are the right-filtrations for the two length-2 types:

$$
\begin{aligned}
& \mathrm{R}\left(V_{1} \stackrel{f_{1}}{\longrightarrow} V_{2}\right)=\left(0, f_{1}\left(V_{1}\right), V_{2}\right), \\
& \mathrm{R}\left(V_{1} \stackrel{g_{1}}{\longleftarrow} V_{2}\right)=\left(0, g_{1}^{-1}(0), V_{2}\right) .
\end{aligned}
$$

Example 3.3 Here are the right-filtrations for the four length-3 types:

$$
\begin{aligned}
& \mathrm{R}\left(V_{1} \stackrel{f_{1}}{\longrightarrow} V_{2} \stackrel{f_{2}}{\longrightarrow} V_{3}\right)=\left(0, f_{2} f_{1}\left(V_{1}\right), f_{2}\left(V_{2}\right), V_{3}\right), \\
& \mathrm{R}\left(V_{1} \stackrel{f_{1}}{\longrightarrow} V_{2} \stackrel{g_{2}}{\longleftarrow} V_{3}\right)=\left(0, g_{2}^{-1}(0), g_{2}^{-1} f_{1}\left(V_{1}\right), V_{3}\right), \\
& \mathrm{R}\left(V_{1} \stackrel{g_{1}}{\longleftarrow} V_{2} \stackrel{f_{2}}{\longrightarrow} V_{3}\right)=\left(0, f_{2} g_{1}^{-1}(0), f_{2}\left(V_{2}\right), V_{3}\right), \\
& \mathrm{R}\left(V_{1} \stackrel{g_{1}}{\longleftarrow} V_{2} \stackrel{g_{2}}{\longleftarrow} V_{3}\right)=\left(0, g_{2}^{-1}(0), g_{2}^{-1} g_{1}^{-1}(0), V_{3}\right) .
\end{aligned}
$$

See Fig. 2 for a schematic representation.

Remark In the examples above, it is not difficult to see that $\mathrm{R}(\mathbb{V})$ comprises all the subspaces of $V_{n}$ that are naturally definable in terms of the maps $p_{i}$.

Each of the $n$ subquotients $R_{i} / R_{i-1}$ carries information dating back to some earliest $V_{j}$ in the sequence of vector spaces.

Example 3.4 The module $V_{1} \stackrel{f_{1}}{\longrightarrow} V_{2}$ has right-filtration $\left(0, f_{1}\left(V_{1}\right), V_{2}\right)$. The first subquotient $f_{1}\left(V_{1}\right) / 0=f_{1}\left(V_{1}\right)$ corresponds to vectors born at time 1 which survive to time 2 . The second subquotient $V_{2} / f_{1}\left(V_{1}\right)$ corresponds to vectors which appear only at time 2 . 

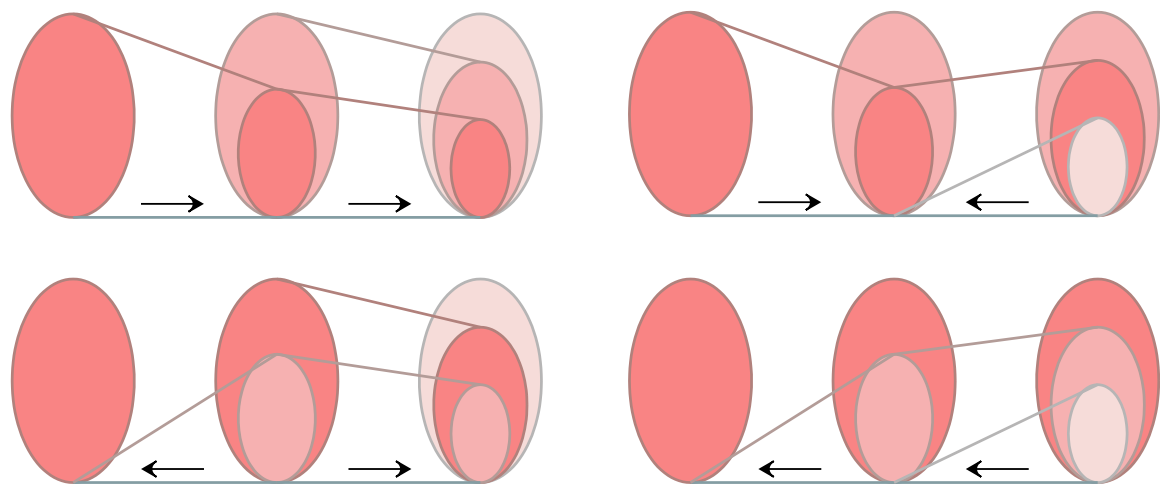

Fig. 2 Forward propagation of the right-filtration, illustrated for the four types $f f, f g, g f, g g$ of length 3

Example 3.5 The module $V_{1} \stackrel{g_{1}}{\longleftarrow} V_{2}$ has right-filtration $\left(0, g_{1}^{-1}(0), V_{2}\right)$. The first subquotient $g_{1}^{-1}(0)$ corresponds to vectors at time 2 which are destroyed when mapping back to time 1 . The second subquotient $V_{2} / g_{1}^{-1}(0)$ is isomorphic to $g_{1}\left(V_{2}\right)$ and records those vectors which survive from time 2 back to time 1 .

Definition 3.6 The birth-time index $\mathrm{b}(\tau)=\left(b_{1}, b_{2}, \ldots, b_{n}\right)$ is a vector of integers $b_{i}$ which indicate the birth times associated with the subquotients $R_{i} / R_{i-1}$ of the right-filtration of a $\tau$-module. This is defined recursively as follows.

Base case:

- If $\tau$ is empty (i.e. $\mathbb{V}$ has length 1 ) then $b(\tau)=(1)$.

Recursive step. Suppose we have already defined $\mathrm{b}(\tau)=\left(b_{1}, b_{2}, \ldots, b_{n}\right)$ :

- If $\tau^{+}$is $\tau f$ then $\mathrm{b}\left(\tau^{+}\right)=\left(b_{1}, \ldots, b_{n}, n+1\right)$.

- If $\tau^{+}$is $\tau g$ then $\mathrm{b}\left(\tau^{+}\right)=\left(n+1, b_{1}, \ldots, b_{n}\right)$.

Example 3.7 At length 2 we have $\mathrm{b}(f)=(1,2)$ whereas $\mathrm{b}(g)=(2,1)$. This is consonant with the discussion in Examples 3.4 and 3.5.

Example 3.8 Here are the birth-time indices for the types of length 3.

$$
\begin{array}{ll}
\mathrm{b}(f f)=(1,2,3), & \mathrm{b}(f g)=(3,1,2), \\
\mathrm{b}(g f)=(2,1,3), & \mathrm{b}(g g)=(3,2,1) .
\end{array}
$$

In summary, the information in a $\tau$-module $\mathbb{V}$ which survives to time $n$ is encoded as a filtration $R(\mathbb{V})$ on $V_{n}$. The 'age' of the information at each level of the filtration (i.e. at each subquotient) is recorded in the birth-time index $b(\tau)$.

For a simplified but precise version of this last claim, we now calculate the rightfiltrations of interval $\tau$-modules. In the filtration specified in the following lemma, $J_{i} / J_{i-1}=\mathrm{k}$ is the only non-zero subquotient, corresponding to the birth time $b_{i}$. 
Lemma 3.9 Let $\tau$ be a type of length $n$, with $\mathrm{b}(\tau)=\left(b_{1}, b_{2}, \ldots, b_{n}\right)$. For each $i=$ $1,2, \ldots, n$, we have an isomorphism

$$
\mathrm{R}\left(\mathbb{I}_{\tau}\left(b_{i}, n\right)\right)=\mathcal{J}(i, n),
$$

where $\mathcal{J}(i, n)=\left(J_{0}, J_{1}, \ldots, J_{n}\right)$ is the filtration on $\mathrm{k}$ defined by

$$
J_{0}=\cdots=J_{i-1}=0 ; \quad J_{i}=\cdots=J_{n}=\mathrm{k} .
$$

Remark We refer to the $\mathcal{J}(b, n)$ also as intervals (but now in the category of filtered vector spaces).

Proof This is a straightforward calculation by induction on $\tau$. For the base case, $\tau$ is empty and $\mathrm{b}(\tau)=(1)$. Then $\mathrm{R}(\mathbb{I}(1,1))=(0, \mathrm{k})=\mathcal{J}(1,1)$ as claimed. Now suppose the result is known for $\tau$, with $\mathrm{b}(\tau)=\left(b_{1}, \ldots, b_{n}\right)$. Suppose $\tau^{+}=\tau f$ or $\tau g$. In both cases, write $\mathrm{b}\left(\tau^{+}\right)=\left(b_{1}^{+}, \ldots, b_{n+1}^{+}\right)$.

Case $f$ : Suppose that $1 \leq i \leq n$; then $b_{i}^{+}=b_{i}$ and therefore

$$
\mathbb{I}_{\tau^{+}}\left(b_{i}^{+}, n+1\right)=\left(\mathbb{I}_{\tau}\left(b_{i}, n\right) \stackrel{1}{\longrightarrow} \mathrm{k}\right) .
$$

Writing $\mathrm{R}\left(\mathbb{I}_{\tau}\left(b_{i}, n\right)\right)=\mathcal{J}(i, n)=\left(J_{0}, J_{1}, \ldots, J_{n}\right)$, it follows that

$$
\mathrm{R}\left(\mathbb{I}_{\tau^{+}}\left(b_{i}^{+}, n+1\right)\right)=\left(J_{0}, J_{1}, \ldots, J_{n}, \mathrm{k}\right)=\mathcal{J}(i, n+1) .
$$

For $i=n+1$, we have $b_{n+1}^{+}=n+1$, and indeed

$$
\begin{aligned}
\mathrm{R}\left(\mathbb{I}_{\tau^{+}}(n+1, n+1)\right) & =\mathrm{R}((0 \longleftrightarrow \cdots \longleftrightarrow 0) \longrightarrow \mathrm{k}) \\
& =(0, \ldots, 0, \mathrm{k}) \\
& =\mathcal{J}(n+1, n+1) .
\end{aligned}
$$

Case $g$ : Suppose that $2 \leq i \leq n+1$; then $b_{i}^{+}=b_{i-1}$ and therefore

$$
\mathbb{I}_{\tau^{+}}\left(b_{i}^{+}, n+1\right)=\left(\mathbb{I}_{\tau}\left(b_{i-1}, n\right) \stackrel{1}{\longleftarrow} \mathrm{k}\right) .
$$

Writing $\mathrm{R}\left(\mathbb{I}_{\tau}\left(b_{i-1}, n\right)\right)=\mathcal{J}(i-1, n)=\left(J_{0}, J_{1}, \ldots, J_{n}\right)$, it follows that

$$
\mathrm{R}\left(\mathbb{I}_{\tau^{+}}\left(b_{i}^{+}, n+1\right)\right)=\left(0, J_{0}, J_{1}, \ldots, J_{n}\right)=\mathcal{J}(i, n+1) .
$$

For $i=1$, we have $b_{1}^{+}=n+1$ and then

$$
\begin{aligned}
\mathrm{R}\left(\mathbb{I}_{\tau^{+}}(n+1, n+1)\right) & =\mathrm{R}((0 \longleftrightarrow \cdots \longleftrightarrow 0) \longleftarrow \mathrm{k}) \\
& =(0, \mathrm{k}, \ldots, \mathrm{k}) \\
& =\mathcal{J}(1, n+1)
\end{aligned}
$$

as required. 
Thus the right-filtration (with the help of the birth-time index) distinguishes the different intervals $\mathbb{I}(b, n)$. It gives no information about intervals $\mathbb{I}(b, d)$ when $d<n$, since in those cases $I_{n}=0$.

Example 3.10 Consider $\tau=f g f$, so $\mathrm{b}(\tau)=\left(b_{1}, b_{2}, b_{3}, b_{4}\right)=(3,1,2,4)$ and in general

$$
\mathrm{R}\left(V_{1} \stackrel{f_{1}}{\longrightarrow} V_{2} \stackrel{g_{2}}{\longleftarrow} V_{3} \stackrel{f_{3}}{\longrightarrow} V_{4}\right)=\left(0, f_{3} g_{2}^{-1}(0), f_{3} g_{2}^{-1} f_{1}\left(V_{1}\right), f_{3}\left(V_{3}\right), V_{4}\right) .
$$

In particular,

$$
\begin{aligned}
& \mathrm{R}\left(\mathbb{I}_{\tau}\left(b_{2}, 4\right)\right)=\mathrm{R}(\mathrm{k} \longrightarrow \mathrm{1} \longrightarrow \stackrel{1}{\mathrm{k}} \mathrm{k} \underset{1}{\longrightarrow} \mathrm{k})=(0,0, \mathrm{k}, \mathrm{k}, \mathrm{k})=\mathcal{J}(2,4), \\
& \mathrm{R}\left(\mathbb{I}_{\tau}\left(b_{3}, 4\right)\right)=\mathrm{R}(0 \longrightarrow \mathrm{k} \leftarrow \mathrm{k} \underset{1}{\longrightarrow} \mathrm{k})=(0,0,0, \mathrm{k}, \mathrm{k})=\mathcal{J}(3,4), \\
& \mathrm{R}\left(\mathbb{I}_{\tau}\left(b_{1}, 4\right)\right)=\mathrm{R}(0 \longrightarrow 0 \longleftarrow \mathrm{k} \longrightarrow \mathrm{1} \longrightarrow \mathrm{k})=(0, \mathrm{k}, \mathrm{k}, \mathrm{k}, \mathrm{k})=\mathcal{J}(1,4), \\
& \mathrm{R}\left(\mathbb{I}_{\tau}\left(b_{4}, 4\right)\right)=\mathrm{R}(0 \longrightarrow 0 \longleftarrow 0 \longrightarrow \mathrm{k})=(0,0,0,0, \mathrm{k})=\mathcal{J}(4,4)
\end{aligned}
$$

which is in accordance with Lemma 3.9.

\subsection{Decompositions of Filtered Vector Spaces}

We now consider filtered vector spaces in their own right, independently of the connection to zigzag modules, and develop the theory of their Remak decompositions. We will see later that this is the right tool for understanding Remak decompositions of zigzag modules.

A filtered vector space of depth $n$ is a sequence $\mathcal{R}=\left(R_{0}, R_{1}, \ldots, R_{n}\right)$ of vector spaces, where $0=R_{0} \leq R_{1} \leq \cdots \leq R_{n}$. The class of such objects is denoted Filt $t_{n}$. The right-filtration $\mathrm{R}(\mathbb{V})$ of any zigzag module $\mathbb{V}$ of length $n$ belongs to this class, as do the intervals $\mathcal{J}(i, n)$ defined in Lemma 3.9. Indeed, if $\mathcal{R} \in \mathrm{Filt}_{n}$ satisfies $\operatorname{dim}\left(R_{n}\right)=1$, then $\mathcal{R}$ is isomorphic to some $\mathcal{J}(i, n)$.

Remark Filt ${ }_{n}$ can be given the structure of a category in a natural way, but it is not quite an abelian category since morphisms do not generally have cokernels.

A filtered vector space $\mathcal{S}=\left(S_{0}, S_{1}, \ldots, S_{n}\right)$ is a subspace of $\mathcal{R}$ if $S_{i} \leq R_{i}$ for all $i$. It is appropriate to consider a stronger notion of subspace when dealing with directsum decompositions: $\mathcal{S}$ is an induced subspace of $\mathcal{R}$ if there exists a vector subspace $K \leq R_{n}$ such that $S_{i}=R_{i} \cap K$ for all $i$. In that event, we write $\mathcal{S}=\mathcal{R} \cap K$. Note that $K=R_{n} \cap K=S_{n}$.

We say that $\mathcal{R}$ is the direct sum of two subspaces, and write $\mathcal{R}=\mathcal{S} \oplus \mathcal{T}$, if $R_{i}=$ $S_{i} \oplus T_{i}$ for all $i$. We claim that $\mathcal{S}, \mathcal{T}$ must be induced subspaces. Note that $S_{n} \cap T_{n}=0$. For each $i$, then, $R_{i} \cap S_{n}$ is a subspace of $R_{i}$ which contains $S_{i}$ and meets $T_{i} \leq T_{n}$ only at 0 . It follows that $R_{i} \cap S_{n}=S_{i}$ for all $i$. Thus $\mathcal{S}=\mathcal{R} \cap S_{n}$, and symmetrically $\mathcal{T}=\mathcal{R} \cap T_{n}$.

The general form of a direct-sum decomposition is therefore $\mathcal{R}=(\mathcal{R} \cap K) \oplus$ $(\mathcal{R} \cap L)$. What are the requirements on $K, L$ to make this a valid decomposition? 
The direct-sum condition implies that $R_{n}=K \oplus L$ as a vector space. Moreover, given a vector space decomposition $R_{n}=K \oplus L$, the further condition

$$
R_{i}=\operatorname{Span}\left(R_{i} \cap K, R_{i} \cap L\right) \quad \text { for all } i
$$

is necessary and sufficient to guarantee $\mathcal{R}=(\mathcal{R} \cap K) \oplus(\mathcal{R} \cap L)$.

If $\mathcal{R}=\mathcal{S} \oplus \mathcal{T}$, the two subspaces $\mathcal{S}, \mathcal{T}$ are said to be complementary summands. The following fact radically simplifies the decomposition theory of filtered vector spaces.

Proposition 3.11 Every induced subspace of a filtered vector space has a complementary summand.

Proof We are given $\mathcal{S}=\mathcal{R} \cap K$, and seek to construct $\mathcal{T}=\left(T_{0}, T_{1}, \ldots, T_{n}\right)$ such that $\mathcal{R}=\mathcal{S} \oplus \mathcal{T}$. We proceed inductively. Since $R_{0}=S_{0}=0$ we take $T_{0}=0$. Now suppose we have chosen $T_{k}$ so that $R_{k}=S_{k} \oplus T_{k}$. In particular, $T_{k} \cap S_{k}=0$. Then

$$
T_{k} \cap S_{k+1} \leq T_{k} \cap S_{n}=\left(T_{k} \cap R_{k}\right) \cap S_{n}=T_{k} \cap\left(R_{k} \cap S_{n}\right)=T_{k} \cap S_{k}=0 .
$$

Thus $T_{k}$ and $S_{k+1}$ are independent subspaces of $R_{k+1}$, and so $T_{k}$ can be extended to a complement $T_{k+1}$ of $S_{k+1}$ in $R_{k+1}$. This completes the induction.

Corollary 3.12 The indecomposables in Filt ${ }_{n}$ are precisely the intervals $\mathcal{J}(i, n)$. Thus, every filtered vector space can be decomposed as a finite direct sum of intervals.

Proof By Proposition 3.11, $\mathcal{R}$ has nontrivial summands if and only if $R_{n}$ has nontrivial vector subspaces; this happens exactly when $\operatorname{dim}\left(R_{n}\right)>1$.

The dimension of $\mathcal{R} \in$ Filt $_{n}$ is defined to be the vector of integers

$$
\operatorname{dim}(\mathcal{R})=\left(c_{1}, c_{2}, \ldots, c_{n}\right)
$$

where $c_{i}=\operatorname{dim}\left(R_{i} / R_{i-1}\right)$ are the dimensions of the successive subquotients of the filtration.

Proposition 3.13 Let $\mathcal{R}$ be a filtered vector space of depth $n$, with $\operatorname{dim}(\mathcal{R})=$ $\left(c_{1}, c_{2}, \ldots, c_{n}\right)$. For any decomposition of $\mathcal{R}$ into intervals, the multiplicity of $\mathcal{J}(i, n)$ is $c_{i}$. Thus:

$$
\mathcal{R} \cong \bigoplus_{1 \leq i \leq n} c_{i} \mathcal{J}(i, n)
$$

Proof Let $m_{i}$ be the multiplicity of $\mathcal{J}(i, n)$. Then, for all $k$,

$$
\operatorname{dim}\left(R_{k}\right)=m_{1}+m_{2}+\cdots+m_{k}
$$

by considering the contribution of each summand, whereas

$$
\operatorname{dim}\left(R_{k}\right)=c_{1}+c_{2}+\cdots+c_{k}
$$


by considering the contribution of each subquotient $R_{i} / R_{i-1}$. This is possible only if $m_{i}=c_{i}$ for all $i$.

This concludes our tour of the decomposition theory for filtered vector spaces. Now we must leverage this to achieve a decomposition theory for $\tau$-modules. In one direction, the relationship is straightforward:

Proposition 3.14 The right-filtration operation respects direct sums, in the sense that

$$
\mathrm{R}\left(\mathbb{V}_{1} \oplus \cdots \oplus \mathbb{V}_{N}\right)=\mathrm{R}\left(\mathbb{V}_{1}\right) \oplus \cdots \oplus \mathrm{R}\left(\mathbb{V}_{N}\right)
$$

for $\tau$-modules $\mathbb{V}_{1}, \ldots, \mathbb{V}_{N}$.

Proof This is proved by induction on $\tau$, following the recursive structure of Definition 3.1 and using the standard facts

$$
\left(f_{1} \oplus \cdots \oplus f_{N}\right)\left(R_{1} \oplus \cdots \oplus R_{N}\right)=f_{1}\left(R_{1}\right) \oplus \cdots \oplus f_{N}\left(R_{N}\right)
$$

and

$$
\left(g_{1} \oplus \cdots \oplus g_{N}\right)^{-1}\left(R_{1} \oplus \cdots \oplus R_{N}\right)=g_{1}^{-1}\left(R_{1}\right) \oplus \cdots \oplus g_{N}^{-1}\left(R_{N}\right)
$$

from linear algebra. (For simplicity we are suppressing various indices here.)

However, what we need is a converse to Proposition 3.14: if the filtered vector space $\mathcal{R}=\mathrm{R}(\mathbb{V})$ can be split as a direct sum $\mathcal{R}=\mathcal{R}_{1} \oplus \cdots \oplus \mathcal{R}_{N}$, we would like to infer a corresponding splitting $\mathbb{V}=\mathbb{V}_{1} \oplus \cdots \oplus \mathbb{V}_{N}$ of $\tau$-modules. In the following two sections we establish such a principle for a particular class: the 'streamlined' $\tau$-modules.

\subsection{Streamlined Modules}

We introduce streamlined modules: a special class of $\tau$-module for which the rightfiltration functor preserves all structural information. A streamlined module can be decomposed into intervals by decomposing its right-filtration (Lemma 3.18). As we will see, the general decomposition theorem (Theorem 4.1) reduces to this special case: an arbitrary zigzag module can be written as a direct sum of streamlined modules of different lengths (Lemma 4.3).

Definition 3.15 A $\tau$-module $\mathbb{V}$ is (right-)streamlined if each $\stackrel{f_{i}}{\longrightarrow}$ is injective and each $\stackrel{g_{i}}{\longleftarrow}$ is surjective.

Similarly, we may say that a $\tau$-module $\mathbb{V}$ is left-streamlined if each $\stackrel{f_{i}}{\longrightarrow}$ is surjective and each $\stackrel{g_{i}}{\longleftarrow}$ is injective. We will not need to consider left-streamlined modules until Sect. 5. By default, 'streamlined' will be taken to mean 'right-streamlined'. 
Example 3.16 Intervals $\mathbb{I}(b, n)$ are streamlined (but not $\mathbb{I}(b, d)$ for $d<n)$. Conversely, a streamlined $\tau$-module $\mathbb{V}$ with $\operatorname{dim}\left(V_{n}\right)=1$ is necessarily isomorphic to some $\mathbb{I}(b, n)$. Indeed, $\operatorname{dim}\left(V_{i}\right)$ is a non-decreasing sequence and therefore comprises some $b-1$ zeros (where $1 \leq b \leq n$ ) followed by $n-b+1$ ones. The maps between the 1-dimensional terms are injective or surjective, and therefore isomorphisms.

Proposition 3.17 A direct sum $\mathbb{V}=\mathbb{V}_{1} \oplus \cdots \oplus \mathbb{V}_{N}$ of $\tau$-modules is streamlined if and only if each summand is streamlined.

Proof Each $\stackrel{f}{\longrightarrow}$ in $\mathbb{V}$ decomposes as $f=f_{1} \oplus \cdots \oplus f_{N}$ and is injective if and only if each $f_{j}$ is injective. Each $\stackrel{g}{\longleftarrow}$ in $\mathbb{V}$ decomposes as $g=g_{1} \oplus \cdots \oplus g_{N}$ and is surjective if and only if each $g_{j}$ is surjective.

The proof of the following lemma appears at the end of this section.

Lemma 3.18 (Decomposition Lemma) Let $\mathbb{V}$ be a streamlined $\tau$-module and let $\mathcal{R}=$ $\mathrm{R}(\mathbb{V})$. For any decomposition $\mathcal{R}=\mathcal{S}_{1} \oplus \cdots \oplus \mathcal{S}_{N}$, there exists a unique decomposition $\mathbb{V}=\mathbb{W}_{1} \oplus \cdots \oplus \mathbb{W}_{N}$ such that $\mathcal{S}_{i}=\mathrm{R}\left(\mathbb{W}_{j}\right)$ for all $j$.

Theorem 3.19 (Interval decomposition for streamlined modules) Let $\mathbb{V}$ be a streamlined $\tau$-module of length $n$, and write $\operatorname{dim}(\mathrm{R}(\mathbb{V}))=\left(c_{1}, c_{2}, \ldots, c_{n}\right)$ and $\mathrm{b}(\tau)=$ $\left(b_{1}, b_{2}, \ldots, b_{n}\right)$. Then there is an isomorphism of $\tau$-modules

$$
\mathbb{V} \cong \bigoplus_{1 \leq i \leq n} c_{i} \mathbb{I}\left(b_{i}, n\right)
$$

Proof Let $\mathcal{R}=\mathrm{R}(\mathbb{V})$. By Proposition 3.13, there is a decomposition $\mathcal{R}=\mathcal{J}_{1} \oplus \cdots \oplus$ $\mathcal{J}_{N}$, where the $\mathcal{J}_{j}$ are a collection of $N=c_{1}+\cdots+c_{n}$ intervals with $\mathcal{J}(i, n)$ occurring with multiplicity $c_{i}$. Lemma 3.18 produces a decomposition $\mathbb{V}=\mathbb{I}_{1} \oplus \cdots \oplus \mathbb{I}_{N}$, with $\mathrm{R}\left(\mathbb{I}_{j}\right)=\mathcal{J}_{j}$ for all $j$. Each $\mathbb{I}_{j}$ is streamlined (Proposition 3.17) with maximum dimension $\operatorname{dim}\left(\left(I_{j}\right)_{n}\right)=1$, and is therefore isomorphic to some $\mathbb{I}(b, n)$ (Example 3.16). By Lemma 3.9, we must have $\mathbb{I}_{j}=\mathbb{I}\left(b_{i}, n\right)$ whenever $\mathcal{J}_{j}=\mathcal{J}(i, n)$. It follows that the $\mathbb{I}_{j}$ are a collection of $N=c_{1}+\cdots+c_{n}$ intervals with $\mathbb{I}\left(b_{i}, n\right)$ occurring with multiplicity $c_{i}$.

We complete this section with a proof of the Decomposition Lemma.

Proof of Lemma 3.18 We may assume that $N=2$, since the general case follows by iteration. Accordingly, suppose that $\mathcal{R}=\mathrm{R}(\mathbb{V})$ can be written in the form $\mathcal{R}=\mathcal{S} \oplus \mathcal{T}$; we must show that there is a corresponding decomposition $\mathbb{V}=\mathbb{W} \oplus \mathbb{X}$. We will argue by induction on $n=$ length $(\tau)$.

The first step is to determine the splitting $V_{n}=W_{n} \oplus X_{n}$. In fact, the stipulation that $\mathcal{S}=\mathrm{R}(\mathbb{W})$ and $\mathcal{T}=\mathrm{R}(\mathbb{X})$ forces $W_{n}=S_{n}$ and $X_{n}=T_{n}$. If $n=1$, then we are done. Otherwise, let $\hat{\mathbb{V}}$ denote the truncation of $\mathbb{V}$ to the indices $\{1, \ldots, n-1\}$ and let $\hat{\mathcal{R}}=\mathrm{R}(\hat{\mathbb{V}})$. We will shortly establish that $\mathcal{R}=\mathcal{S} \oplus \mathcal{T}$ induces a unique compatible decomposition $\hat{\mathcal{R}}=\hat{\mathcal{S}} \oplus \hat{\mathcal{T}}$. The inductive hypothesis will then provide $\hat{\mathbb{V}}=\widehat{\mathbb{W}} \oplus \hat{\mathbb{X}}$, 
which combines with $V_{n}=W_{n} \oplus X_{n}$ to produce the desired decomposition $\mathbb{V}=$ $\mathbb{W} \oplus \mathbb{X}$. That will complete the proof.

Write $\mathcal{R}=\left(R_{0}, R_{1}, \ldots, R_{n}\right)$. There are two cases.

Case $\stackrel{f_{n-1}}{\longrightarrow}$, injective. We can identify $V_{n-1}$ with the subspace $f_{n-1}\left(V_{n-1}\right)=R_{n-1}$ of $V_{n}$. Thereupon we have

$$
\hat{\mathcal{R}}=\left(R_{0}, R_{1}, \ldots, R_{n-1}\right) .
$$

The unique splitting of $V_{n-1}$ compatible with $V_{n}=W_{n} \oplus X_{n}$ is

$$
V_{n-1}=\left(R_{n-1} \cap W_{n}\right) \oplus\left(R_{n-1} \cap X_{n}\right)=S_{n-1} \oplus T_{n-1} .
$$

We must now verify that the induced subspaces $\hat{\mathcal{S}}=\hat{\mathcal{R}} \cap S_{n-1}$ and $\hat{\mathcal{T}}=\hat{\mathcal{R}} \cap T_{n-1}$ give a valid decomposition $\hat{\mathcal{R}}=\hat{\mathcal{S}} \oplus \hat{\mathcal{T}}$ of filtered vector spaces. This follows because $\hat{S}_{i}=R_{i} \cap S_{n-1}=R_{i} \cap S_{n}=S_{i}$ and similarly $\hat{T}_{i}=T_{i}$, for all $i<n$; so $R_{i}=S_{i} \oplus T_{i}=$ $\hat{S}_{i} \oplus \hat{T}_{i}$ as required.

Case $\stackrel{g_{n-1}}{\longleftarrow}$, surjective. Here we identify $V_{n-1}$ as the quotient $V_{n} / \operatorname{ker}\left(g_{n-1}\right)=$ $R_{n} / R_{1}$. Under this identification,

$$
\hat{\mathcal{R}}=\left(R_{1} / R_{1}, R_{2} / R_{1}, \ldots, R_{n} / R_{1}\right) .
$$

In splitting $V_{n-1}=W_{n-1} \oplus X_{n-1}$ we are compelled to take

$$
W_{n-1}=g_{n-1}\left(W_{n}\right)=S_{n} / S_{1}, \quad X_{n-1}=g_{n-1}\left(X_{n}\right)=T_{n} / T_{1},
$$

which induce

$$
\hat{S}_{i}=g_{n-1}\left(S_{i+1}\right)=S_{i+1} / S_{1}, \quad \hat{T}_{i}=g_{n-1}\left(T_{i+1}\right)=T_{i+1} / T_{1},
$$

for the purported splitting $\hat{\mathcal{R}}=\hat{\mathcal{S}} \oplus \hat{\mathcal{T}}$. To confirm that this is a genuine decomposition, we note from linear algebra that the twin facts

$$
R_{i+1}=S_{i+1} \oplus T_{i+1}, \quad R_{1}=S_{1} \oplus T_{1}=\left(S_{i+1} \cap R_{1}\right) \oplus\left(T_{i+1} \cap R_{1}\right)
$$

imply that

$$
R_{i+1} / R_{1}=\left(S_{i+1} / S_{1}\right) \oplus\left(T_{i+1} / T_{1}\right)
$$

as required.

Remark There is a high-level proof of Lemma 3.18 which in some sense is the natural explanation for the result. We outline this proof now. The first observation is that the transformation $\mathbb{V} \rightarrow \mathrm{R}(\mathbb{V})$ is a functor from $\tau$ Mod to Filt $_{n}$ : a morphism $\alpha: \mathbb{V} \rightarrow \mathbb{W}$ induces a morphism $\mathrm{R}(\alpha): \mathrm{R}(\mathbb{V}) \rightarrow \mathrm{R}(\mathbb{W})$. Indeed, $\mathrm{R}(\alpha)$ is defined to be $\alpha_{n}: V_{n} \rightarrow W_{n}$; one must check that this respects the filtrations on $V_{n}$ and $W_{n}$. Being a functor, $\mathrm{R}$ defines a ring homomorphism $\operatorname{End}(\mathbb{V}) \rightarrow \operatorname{End}(\mathrm{R}(\mathbb{V}))$. The second key fact is that this homomorphism is an isomorphism if $\mathbb{V}$ is streamlined (in general it is surjective). It is well known that direct-sum decompositions of a module can be extracted from the structure of its endomorphism ring: direct summands correspond to idempotent elements of the ring. It follows that $\mathbb{V}$ and $R(\mathbb{V})$ have the same decomposition structure. 


\section{The Interval Decomposition Algorithm}

Here we describe the algorithm for determining the indecomposable factors of a $\tau$ module. We give three versions of the 'algorithm'.

The first version, in Sect. 4.1, is not an algorithm but a proof that every $\tau$-module decomposes as a sum of interval modules (Theorem 2.5). Moreover, the structure of the proof makes it clear how to compute the interval decomposition (Theorem 4.1). The algorithms in the subsequent sections build on this.

In Sect. 4.2 we describe an abstract form of the decomposition algorithm, using the language of vector spaces and linear maps. No consideration is given to how the spaces and maps are described and manipulated in practice.

In Sect. 4.3 we suppose that the maps $f_{i}, g_{i}$ are presented concretely as matrices $M_{i}, N_{i}$ with respect to a choice of bases for the vector spaces $V_{i}$. We describe an algorithm which takes these matrices as input and returns the interval decomposition.

\subsection{The Interval Decomposition Theorem}

Our present goal is to give a somewhat constructive proof of Theorem 2.5, which asserts that any $\tau$-module $\mathbb{V}$ is isomorphic to a direct sum of intervals $\mathbb{I}(b, d)$. We prove a stronger, more precise result, which explicitly determines the multiplicity of each interval within $\mathbb{V}$.

Some notation will help with the theorem statement. If

$$
\mathbb{V}=\left(V_{1} \stackrel{p_{1}}{\longleftrightarrow} \cdots \stackrel{p_{n-1}}{\longleftrightarrow} V_{n}\right)
$$

then let

$$
\mathbb{V}[k]=\left(V_{1} \stackrel{p_{1}}{\longleftrightarrow} \cdots \stackrel{p_{k-1}}{\longleftrightarrow} V_{k}\right)
$$

denote the truncation of $\mathbb{V}$ to length $k$, and let $\tau[k]$ denote its type (which is a truncation of $\tau$ ).

Theorem 4.1 (Interval decomposition) Let $\mathbb{V}$ be a $\tau$-module. For $1 \leq k \leq n$, define

$$
\left(b_{1}^{k}, b_{2}^{k}, \ldots, b_{k}^{k}\right)=\mathrm{b}(\tau[k]) .
$$

Writing $\mathcal{R}_{k}=\mathrm{R}(\mathbb{V}[k])$, define

$$
\left(c_{1}^{k}, c_{2}^{k}, \ldots, c_{k}^{k}\right)=\left\{\begin{array}{l}
\operatorname{dim}\left(\mathcal{R}_{k} \cap \operatorname{Ker}\left(f_{k}\right)\right), \\
\operatorname{dim}\left(\mathcal{R}_{k}\right)-\operatorname{dim}\left(\mathcal{R}_{k} \cap \operatorname{Im}\left(g_{k}\right)\right)
\end{array}\right.
$$

(whichever is applicable) when $k \neq n$, and

$$
\left(c_{1}^{n}, c_{2}^{n}, \ldots, c_{n}^{n}\right)=\operatorname{dim}\left(\mathcal{R}_{n}\right) .
$$

Then

$$
\mathbb{V} \cong \bigoplus_{1 \leq i \leq k \leq n} c_{i}^{k} \mathbb{I}\left(b_{i}^{k}, k\right)
$$


Addendum 4.2 In the situation of Theorem 4.1, write

$$
\left(r_{1}^{k}, \ldots, r_{k}^{k}\right)=\operatorname{dim}\left(\mathcal{R}_{k}\right)
$$

for $k=1, \ldots, n$, and conventionally define $r_{i}^{n+1}=0$ for all $i$. Then

$$
c_{i}^{k}= \begin{cases}r_{i}^{k}-r_{i}^{k+1} & \text { case } \stackrel{f_{k}}{\longrightarrow} \\ r_{i}^{k}-r_{i+1}^{k+1} & \text { case } \stackrel{g_{k}}{\longleftarrow}\end{cases}
$$

for $1 \leq i \leq k \leq n$.

The decomposition strategy begins with the following lemma. The idea is to proceed from left to right along the complex, removing streamlined summands at each step. Having done this, the Remak decompositions of those summands can be determined by counting dimensions, as prescribed in Theorem 3.19.

Lemma 4.3 Let $\mathbb{V}=V_{1} \stackrel{p_{1}}{\longleftrightarrow} \cdots \stackrel{p_{n-1}}{\longleftrightarrow} V_{n}$ be an irreducible $\tau$-module of length $n$. Then there exists a direct-sum decomposition

$$
\mathbb{V}=\mathbb{V}^{1} \oplus \mathbb{V}^{2} \oplus \cdots \oplus \mathbb{V}^{n}
$$

where each $\mathbb{V}^{k}$ is supported over the indices $\{1,2, \ldots, k\}$ and is right-streamlined over that range.

The following picture illustrates the decomposition.

$$
\mathbb{V}=\left\{\begin{array}{l}
\mathbb{V}^{1}=V_{1}^{1} \\
\oplus \\
\mathbb{V}^{2}=V_{1}^{2} \stackrel{p_{1}}{\longleftrightarrow} V_{2}^{2} \\
\oplus \\
\mathbb{V}^{3}=V_{1}^{3} \stackrel{p_{1}}{\longleftrightarrow} V_{2}^{3} \stackrel{p_{2}}{\longleftrightarrow} V_{3}^{3} \\
\oplus \\
\vdots \\
\oplus \\
\mathbb{V}^{n}=V_{1}^{n} \stackrel{p_{1}}{\longleftrightarrow} V_{2}^{n} \stackrel{p_{2}}{\longleftrightarrow} V_{3}^{n} \stackrel{p_{3}}{\longleftrightarrow} \cdots \stackrel{p_{n-1}}{\longleftrightarrow} V_{n}^{n}
\end{array}\right.
$$

Each row (i.e. summand) is right-streamlined, and therefore amenable to analysis via the right-filtration functor.

Proof We proceed by induction on the length of $\mathbb{V}$. The inductive statement is that

$$
\mathbb{V}[k]=\mathbb{V}^{1} \oplus \cdots \oplus \mathbb{V}^{k-1} \oplus \mathbb{W}^{k}
$$

where the $\mathbb{V}^{i}$ are as in the theorem statement, and $\mathbb{W}^{k}$ is itself right-streamlined. 
For the base case $k=1$, there is nothing to prove: take $\mathbb{W}^{1}=\mathbb{V}[1]$. Now suppose the inductive statement is established for $k$, and consider $\mathbb{V}[k+1]$. This can be written

$$
\begin{aligned}
V[k+1] & =\left(\mathbb{V}^{1} \oplus \cdots \oplus \mathbb{V}^{k-1} \oplus \mathbb{W}^{k}\right) \stackrel{p_{k}}{\longleftrightarrow} V_{k+1} \\
& =\mathbb{V}^{1} \oplus \cdots \oplus \mathbb{V}^{k-1} \oplus\left(\mathbb{W}^{k} \stackrel{p_{k}}{\longleftrightarrow} V_{k+1}\right),
\end{aligned}
$$

where the rebracketing is permissible because all of the $\mathbb{V}^{i}$ terms terminate before

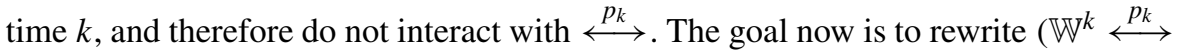
$\left.V_{k+1}\right)$ as $\mathbb{V}^{k} \oplus \mathbb{W}^{k+1}$, where $\mathbb{V}^{k}$ terminates at time $k$ and both $\mathbb{V}^{k}$ and $\mathbb{W}^{k+1}$ are right-streamlined. The rightmost term of $\mathbb{W}^{k}$ is $V_{k}$, so $\mathrm{R}\left(\mathbb{W}^{k}\right)$ is a filtration on $V_{k}$.

Case $f: \mathbb{W}^{k} \stackrel{f_{k}}{\longrightarrow} V_{k+1}$. In other words $f_{k}: V_{k} \rightarrow V_{k+1}$. Let $\mathcal{S}=\mathrm{R}\left(\mathbb{W}^{k}\right) \cap \operatorname{Ker}\left(f_{k}\right)$. Proposition 3.11 implies that $\mathcal{S}$ has a complement in $\mathrm{R}\left(\mathbb{W}^{k}\right)$; say $\mathrm{R}\left(\mathbb{W}^{k}\right)=\mathcal{S} \oplus \mathcal{T}$. This corresponds (Lemma 3.18) to a direct-sum decomposition $\mathbb{W}^{k}=\mathbb{V}^{k} \oplus \hat{\mathbb{W}}^{k}$, where both summands are streamlined (Proposition 3.17). This defines $\mathbb{V}^{k}$, and we set $\mathbb{W}^{k+1}=\left(\hat{\mathbb{W}}^{k} \stackrel{f_{k}}{\longrightarrow} V_{k+1}\right)$. To check that this works, note that $f_{k}$ is zero on $\left(\mathbb{V}^{k}\right)_{k}=\operatorname{Ker}\left(f_{k}\right)$ and is injective on the complementary subspace $\left(\hat{\mathbb{W}}^{k}\right)_{k}$. Thus $\mathbb{V}^{k}$ is a summand of $\mathbb{V}[k+1]$ terminating at time $k$, and $\mathbb{W}^{k+1}$ is streamlined.

Case $g: \mathbb{W}^{k} \stackrel{g_{k}}{\longleftarrow} V_{k+1}$. In other words $g_{k}: V_{k+1} \rightarrow V_{k}$. Let $\mathcal{S}=\mathrm{R}\left(\mathbb{W}^{k}\right) \cap \operatorname{Im}\left(g_{k}\right)$. Proposition 3.11 implies that $\mathcal{S}$ has a complement in $\mathrm{R}\left(\mathbb{W}^{k}\right)$; say $\mathrm{R}\left(\mathbb{W}^{k}\right)=\mathcal{S} \oplus \mathcal{T}$. This corresponds (Lemma 3.18) to a direct-sum decomposition $\mathbb{W}^{k}=\hat{\mathbb{W}}^{k} \oplus \mathbb{V}^{k}$, where both summands are streamlined (Proposition 3.17). This defines $\mathbb{V}^{k}$, and we set $\mathbb{W}^{k+1}=\left(\hat{\mathbb{W}}^{k} \stackrel{g_{k}}{\longleftarrow} V_{k+1}\right)$. To check that this works, note that $g_{k}$ is surjective onto $\left(\hat{\mathbb{W}}^{k}\right)_{k}=\operatorname{Im}\left(g_{k}\right)$ and misses the complementary subspace $\left(\mathbb{V}^{k}\right)_{k}$. Thus $\mathbb{V}^{k}$ is a summand of $\mathbb{V}[k+1]$ terminating at time $k$, and $\mathbb{W}^{k+1}$ is streamlined.

This establishes the inductive step, so eventually

$$
\mathbb{V}=\mathbb{V}[n]=\mathbb{V}^{1} \oplus \cdots \oplus \mathbb{V}^{n-1} \oplus \mathbb{W}^{n}
$$

and we set $\mathbb{V}^{n}=\mathbb{W}^{n}$ to finish the proof.

Proof of Theorem 4.1 Write $\mathbb{V}=\mathbb{V}^{1} \oplus \cdots \oplus \mathbb{V}^{n}$ according to Lemma 4.3. We now calculate the decomposition of each $\mathbb{V}^{k}$ into intervals $\mathbb{I}(b, k)$. Note that

$$
\mathbb{V}[k]=\mathbb{V}^{k} \oplus \mathbb{V}^{k+1}[k] \oplus \cdots \oplus \mathbb{V}^{n}[k] .
$$

We can write $\mathbb{W}^{k}=\mathbb{V}^{k+1} \oplus \cdots \oplus \mathbb{V}^{n}$, so then

$$
\mathcal{R}_{k}=\mathrm{R}\left(\mathbb{V}^{k} \oplus \mathbb{W}^{k}[k]\right)=\mathrm{R}\left(\mathbb{V}^{k}\right) \oplus \mathrm{R}\left(\mathbb{W}^{k}[k]\right)
$$

(using Proposition 3.14). This is a filtration on $V_{k}^{k} \oplus W_{k}^{k}$.

Suppose $k<n$. We note that $\mathbb{W}^{k}$ is streamlined up to time $k+1$, whereas $\mathbb{V}^{k}$ is zero at time $k+1$. The next map in the sequence is

$$
V_{k}^{k} \oplus W_{k}^{k} \stackrel{f_{k}}{\longrightarrow} W_{k+1}^{k} \quad \text { or } \quad V_{k}^{k} \oplus W_{k}^{k} \stackrel{g_{k}}{\longleftarrow} W_{k+1}^{k} \text {. }
$$


In the first case, it follows that $V_{k}^{k}=\operatorname{Ker}\left(f_{k}\right)$ and therefore $\mathrm{R}\left(\mathbb{V}^{k}\right)=\mathcal{R}_{k} \cap \operatorname{Ker}\left(f_{k}\right)$. In the second case, $V_{k}^{k}$ is a complement to $\operatorname{Im}\left(g_{k}\right)$ in $V_{k}$, so $\mathcal{R}_{k}=\mathrm{R}\left(\mathbb{V}^{k}\right) \oplus\left(\mathcal{R}_{k} \cap\right.$ $\left.\operatorname{Im}\left(g_{k}\right)\right)$. Thus

$$
\operatorname{dim}\left(\mathrm{R}\left(\mathbb{V}^{k}\right)\right)=\left\{\begin{array}{l}
\operatorname{dim}\left(\mathcal{R}_{k} \cap \operatorname{Ker}\left(f_{k}\right)\right) \\
\operatorname{dim}\left(\mathcal{R}_{k}\right)-\operatorname{dim}\left(\mathcal{R}_{k} \cap \operatorname{Im}\left(g_{k}\right)\right)
\end{array}\right\}=\left(c_{1}^{k}, \ldots, c_{k}^{k}\right)
$$

(whichever middle term is applicable). When $k=n$, moreover, we have

$$
\operatorname{dim}\left(\mathrm{R}\left(\mathbb{V}^{n}\right)\right)=\operatorname{dim}\left(\mathcal{R}_{n}\right)=\left(c_{1}^{n}, \ldots, c_{n}^{n}\right) .
$$

Thus, at last,

$$
\mathbb{V}=\bigoplus_{1 \leq k \leq n} \mathbb{V}^{k} \cong \bigoplus_{1 \leq k \leq n}\left\{\bigoplus_{1 \leq i \leq k} c_{i}^{k} \mathbb{I}\left(b_{i}^{k}, k\right)\right\}
$$

using Theorem 3.19 to decompose the $\mathbb{V}^{k}$.

Proof of Addendum 4.2 Write $\left(w_{1}^{k}, \ldots, w_{k}^{k}\right)=\operatorname{dim}\left(\mathrm{R}\left(\mathbb{W}^{k}[k]\right)\right)$. Since $\mathcal{R}_{k}=\mathrm{R}\left(\mathbb{V}^{k}\right) \oplus$ $\mathrm{R}\left(\mathbb{W}^{k}[k]\right)$ we can take dimensions and obtain the formula

$$
\left(r_{1}^{k}, \ldots, r_{k}^{k}\right)=\left(c_{1}^{k}, \ldots, c_{k}^{k}\right)+\left(w_{1}^{k}, \ldots, w_{k}^{k}\right) .
$$

Note also that $\mathcal{R}_{k+1}=\mathrm{R}(\mathbb{V}[k+1])=\mathrm{R}\left(\mathbb{W}^{k}[k+1]\right)$. Moreover, $\mathbb{W}^{k}$ is streamlined up to time $k+1$. It follows that

$$
\left(r_{1}^{k+1}, \ldots, r_{k+1}^{k+1}\right)=\operatorname{dim}\left(\mathrm{R}\left(\mathbb{W}^{k}[k+1]\right)\right)= \begin{cases}\left(w_{1}^{k}, \ldots, w_{k}^{k}, ?\right) & \text { case } f \\ \left(?, w_{1}^{k}, \ldots, w_{k}^{k}\right) & \text { case } g\end{cases}
$$

and therefore

$$
c_{i}^{k}=r_{i}^{k}-w_{i}^{k}= \begin{cases}r_{i}^{k}-r_{i}^{k+1} & \text { case } f \\ r_{i}^{k}-r_{i+1}^{k+1} & \text { case } g\end{cases}
$$

which is the desired formula.

\subsection{Abstract Vector Spaces}

We now transcribe Theorem 4.1 as an abstract algorithm for determining the interval structure of a $\tau$-module $\mathbb{V}$ of length $n$. This algorithm will serve as a skeleton for the more concrete algorithms developed later.

Algorithm 4.4 We proceed through $k=1,2, \ldots, n$, computing the filtration $\mathcal{R}_{k}=$ $\mathrm{R}(\mathbb{V}[k])$, the birth-time index $\mathrm{b}(\tau[k])$, and the dimensions $c_{i}^{k}$ iteratively.

BEGIN

Initialisation $(k=1)$ :

(1) $\mathcal{R}_{1}=\left(0, V_{1}\right)$.

(2) $\mathrm{b}(\tau[1])=(1)$. 
Iterative step $(k=1,2, \ldots, n-1)$ :

(3) Calculate $\mathcal{R}_{k+1}$ from $\mathcal{R}_{k}=\left(R_{0}^{k}, R_{1}^{k}, \ldots, R_{k}^{k}\right)$ using Definition 3.1:

$$
\left(R_{0}^{k+1}, R_{1}^{k+1}, \ldots, R_{k+1}^{k+1}\right)= \begin{cases}\left(f_{k}\left(R_{0}^{k}\right), f_{k}\left(R_{1}^{k}\right), \ldots, f_{k}\left(R_{k}^{k}\right), V_{k+1}\right) & \text { case } f \\ \left(0, g_{k}^{-1}\left(R_{0}^{k}\right), g_{k}^{-1}\left(R_{1}^{k}\right), \ldots, g_{k}^{-1}\left(R_{k}^{k}\right)\right) & \text { case } g\end{cases}
$$

(4) Calculate $\mathrm{b}(\tau[k+1]))$ from $\mathrm{b}(\tau[k])=\left(b_{1}^{k}, b_{2}^{k}, \ldots, b_{k}^{k}\right)$ using Definition 3.6:

$$
\left(b_{1}^{k+1}, \ldots, b_{k+1}^{k+1}\right)= \begin{cases}\left(b_{1}^{k}, \ldots, b_{k}^{k}, k+1\right) & \text { case } f \\ \left(k+1, b_{1}^{k}, \ldots, b_{k}^{k}\right) & \text { case } g\end{cases}
$$

(5) Calculate $\left(c_{1}^{k}, \ldots, c_{k}^{k}\right)$ using the formula in Theorem 4.1:

$$
\left(c_{1}^{k}, c_{2}^{k}, \ldots, c_{k}^{k}\right)= \begin{cases}\operatorname{dim}\left(\mathcal{R}_{k} \cap \operatorname{Ker}\left(f_{k}\right)\right) & \text { case } f \\ \operatorname{dim}\left(\mathcal{R}_{k}\right)-\operatorname{dim}\left(\mathcal{R}_{k} \cap \operatorname{Im}\left(g_{k}\right)\right) & \text { case } g\end{cases}
$$

Alternatively, use the formula in Addendum 4.2:

$$
c_{i}^{k}= \begin{cases}r_{i}^{k}-r_{i}^{k+1} & \text { case } f \\ r_{i}^{k}-r_{i+1}^{k+1} & \text { case } g\end{cases}
$$

Here $\left(r_{1}^{k}, \ldots, r_{k}^{k}\right)=\operatorname{dim}\left(\mathcal{R}_{k}\right)$.

Terminal step $(k=n)$ :

(6) Calculate $\left(c_{1}^{n}, \ldots, c_{n}^{n}\right)=\operatorname{dim}(\mathcal{R}(\mathbb{V}))$.

\section{Print results:}

(7) For $1 \leq i \leq k \leq n$, the interval $\mathbb{I}\left(b_{i}^{k}, k\right)$ occurs with multiplicity $c_{i}^{k}$.

END

Note that steps (3-5) have an ' $f$ ' version and a ' $g$ ' version, depending on the direction of the map $p_{k}$.

This abstract algorithm does not specify how the filtered vector spaces $\mathrm{R}(\mathbb{V}[k+1])$ are stored, nor how the maps $f_{k}$ or $g_{k}$ (which are used in steps (3) and (5)) are represented. In any concrete setting, it is necessary to specify data structures. A good choice will facilitate the calculations in steps (3) and (5). In the next section, we work out the details in a simple scenario.

\subsection{Concrete Vector Spaces}

In this section we describe an algorithm to solve the following concrete problem. Let $\tau$ be a type of length $n$. We specify a $\tau$-module $\mathbb{V}$ as follows. Set $V_{i}=\mathrm{k}^{a_{i}}$ for integers $a_{i} \geq 0$. For each $i$, the map $f_{i}$ is defined by an $a_{i+1}$-by- $a_{i}$ matrix $M_{i}$ or else the map $g_{i}$ is defined by an $a_{i}$-by- $a_{i+1}$ matrix $N_{i}$. We are to determine Pers $(\mathbb{V})$, given $\tau$ and the matrices $M_{i}$ or $N_{i}$. 
We follow Algorithm 4.4. The substantial task is to calculate the sequence of rightfiltrations $\mathcal{R}_{k}=\mathrm{R}(\mathbb{V}[k])$, for step (3). Everything else is book-keeping: the birth-time indices $b_{i}^{k}$ are calculated according to step (4); and the filtration dimensions $r_{i}^{k}$ (and hence the $c_{i}^{k}$ ) will be easy to read off from the stored description of the filtrations.

Basis Transformations The algorithm operates on two levels. On the conceptual level, we proceed by modifying the bases of the spaces $V_{i}$ by elementary basis transformations. Initially each basis $\mathcal{B}_{i}$ is the standard basis of $\mathrm{k}^{a_{i}}$. We perform modifications on $\mathcal{B}_{2}, \mathcal{B}_{3}, \ldots, \mathcal{B}_{n-1}$ in sequence. On the pragmatic level, what we actually do is apply elementary row and column operations to the matrices $M_{i}$ or $N_{i}$. We make no attempt to track the bases themselves; instead we implement the effect of those changes on the matrices.

Suppose we apply elementary basis transformations to $\mathcal{B}_{k+1}$ on the conceptual level. On the pragmatic level, we must perform

$$
\text { row operations on } M_{k} \text { or column operations on } N_{k}
$$

and simultaneously perform

$$
\text { column operations on } M_{k+1} \text { or row operations on } N_{k+1}
$$

to enact those transformations. Thus, at every stage we must make parallel changes to two matrices simultaneously. Usually we are working to put $M_{k}$ or $N_{k}$ in a particular form, and while doing so the changes have to be mirrored in $M_{k+1}$ or $N_{k+1}$ (paying no attention yet to the structure of that matrix).

We now make this precise. The elementary transformation $E_{i}(p, q, \lambda)$ is defined as follows. On the conceptual level, this is a modification of $\mathcal{B}_{i}=\left(\beta_{1}, \ldots, \beta_{a_{i}}\right)$ involving basis vectors $\beta_{p}$ and $\beta_{q}$ :

$$
\begin{aligned}
& \beta_{p} \leftarrow \beta_{p}, \\
& \beta_{q} \leftarrow \beta_{q}+\lambda \beta_{p} .
\end{aligned}
$$

On the pragmatic level, if $L$ is a matrix representing a linear map $V_{i} \rightarrow W$ for some $W$ (this will be $N_{i-1}$ or $M_{i}$ in our situation), then we modify the columns of $L$ accordingly:

$$
\begin{aligned}
& \text { Column }_{p} \leftarrow \text { Column }_{p}, \\
& \text { Column }_{q} \leftarrow \text { Column }_{q}+\lambda \text { Column }_{p} .
\end{aligned}
$$

Else, if $L$ represents a linear map of the form $W \rightarrow V_{i}$ (this will be $M_{i-1}$ or $N_{i}$ in our situation) then we must apply the dual transformation to the rows of $L$ :

$$
\begin{aligned}
& \operatorname{Row}_{p} \leftarrow \operatorname{Row}_{p}-\lambda \operatorname{Row}_{q}, \\
& \operatorname{Row}_{q} \leftarrow \operatorname{Row}_{q} .
\end{aligned}
$$

In spirit, we right-multiply by the matrix $\left[\begin{array}{ll}1 & \lambda \\ 0 & 1\end{array}\right]$ to modify columns, or else leftmultiply by the inverse matrix $\left[\begin{array}{cc}1 & -\lambda \\ 0 & 1\end{array}\right]$ to modify rows. 
Besides the elementary transformations $E_{i}(p, q, \lambda)$, it is sometimes appropriate to permute the basis elements. The operation $P_{i}(p, q)$ of interchanging $\beta_{p}$ with $\beta_{q}$ is realised pragmatically by interchanging Column $p$ with $\operatorname{Column}_{q}$, or Row $p$ with $\operatorname{Row}_{q}$, as appropriate.

Filtrations The filtration $\mathcal{R}_{k}=\mathrm{R}(\mathbb{V}[k])$ on $V_{k}$ is to be represented as follows. We require the basis $\mathcal{B}_{k}=\left(\beta_{1}, \ldots, \beta_{a_{i}}\right)$ to be compatible with the filtration, in a sense that will become clear. Assuming such a basis, the filtration $\mathcal{R}_{k}=\left(R_{0}, R_{1}, \ldots, R_{k}\right)$ is represented as a non-decreasing function

$$
\phi_{k}:\left\{1,2, \ldots, a_{i}\right\} \rightarrow\{1, \ldots, k\}
$$

so that

$$
R_{i}=\operatorname{Span}\left\{\beta_{p} \mid \phi_{k}(p) \leq i\right\}
$$

for $i=1, \ldots, k$. In other words: the first few basis elements (those $\beta_{p}$ with $\left.\phi_{k}(p)=1\right)$ form a basis for $R_{1}$; the next few basis elements extend this to a basis for $R_{2}$, and so on. The dimension $r_{i}^{k}=\operatorname{dim}\left(R_{i} / R_{i-1}\right)$ can be read off as the cardinality of $\phi_{k}^{-1}(i)$.

Gaussian Elimination Step (3) boils down to the following task. Suppose that $\mathcal{B}_{k}$ and $\phi_{k}$ together represent the filtration $\mathcal{R}_{k}$; then modify $\mathcal{B}_{k+1}$ and determine $\phi_{k+1}$ to represent $\mathcal{R}_{k+1}$. We now explain how to do this.

Case $M$ : the matrix $M_{k}$ represents a linear map $V_{k} \rightarrow V_{k+1}$. We assume that $\mathcal{B}_{k}$ is compatible with the filtration $\mathcal{R}_{k}$, and that $\phi_{k}$ identifies the filtration. This gives a block structure

$$
M_{k}=\left[\begin{array}{llll}
K_{1} & K_{2} & \cdots & K_{k}
\end{array}\right],
$$

where $K_{i}$ gathers together the columns $q$ with $\phi_{k}(q)=i$. Using row operations only, put $M_{k}$ into (unreduced) row echelon form. This means:

- Each of the top $r$ rows contains a 1 (the pivot) as its leftmost nonzero entry.

- Each pivot lies strictly to the left of the pivots of the rows below it.

- The lowest $a_{k+1}-r$ rows are entirely zero.

These row operations correspond to elementary operations $E_{k+1}(p, q, \lambda)$, and the effect of these operations is felt on the next matrix $M_{k+1}$ or $N_{k+1}$, which must be modified accordingly. We now define $\phi_{k+1}$ as follows:

$$
\phi_{k+1}(p)= \begin{cases}\phi_{k}(q) & \text { if row } p \text { has a pivot in column } q \\ k+1 & \text { if row } p \text { has no pivot. }\end{cases}
$$

See Fig. 3. It is evident in the figure that $R_{i}^{k}$ maps onto $R_{i}^{k+1}$ for all $i$.

Case $N$ : the matrix $N_{k}$ represents a linear map $V_{k+1} \rightarrow V_{k}$. We assume that $\mathcal{B}_{k}$ is compatible with the filtration $\mathcal{R}_{k}$, and that $\phi_{i}$ identifies the filtration. This time we 
Fig. 3 Using row echelon form to compute $\mathcal{R}_{k+1}$ from $\mathcal{R}_{k}$
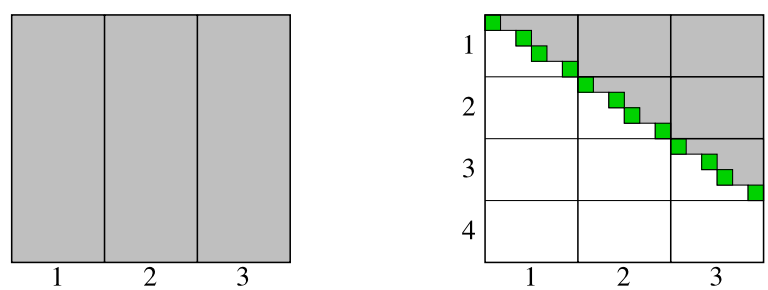

Fig. 4 Using column echelon form to compute $\mathcal{R}_{k+1}$ from $\mathcal{R}_{k}$

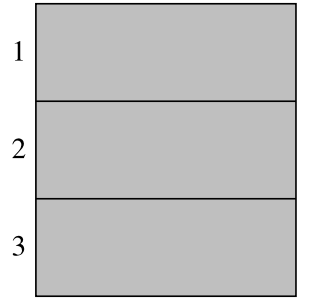

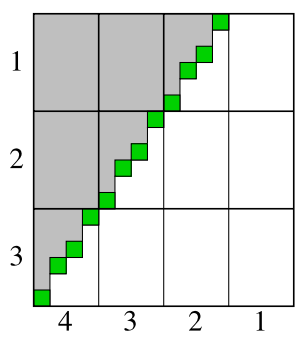

have a vertical block structure

$$
N_{k}=\left[\begin{array}{c}
L_{1} \\
L_{2} \\
\vdots \\
L_{k}
\end{array}\right] \text {, }
$$

where $L_{i}$ gathers together the rows $q$ with $\phi_{k}(q)=i$. Using column operations only, put $N_{k}$ into the column echelon form defined as follows (this echelon form begins on the bottom left):

- Each of the leftmost $r$ columns contains a 1 (the pivot) as its lowest nonzero entry.

- Each pivot lies strictly lower than the pivots of the columns to the right of it.

- The rightmost $a_{k+1}-r$ rows are entirely zero.

These column operations correspond to elementary operations $E_{k+1}(p, q, \lambda)$, and the effect of these operations is felt on the next matrix $M_{k+1}$ or $N_{k+1}$, which must be modified accordingly. We now define $\phi_{k+1}$ as follows:

$$
\phi_{k+1}(p)= \begin{cases}\phi_{k}(q)+1 & \text { if column } p \text { has a pivot in row } q \\ 1 & \text { if column } p \text { has no pivot. }\end{cases}
$$

See Fig. 4. It is evident in the figure that $R_{i+1}^{k+1}$ is the largest subspace which maps into $R_{i}^{k}$, for all $i$.

This concludes our treatment of the concrete form of the zigzag algorithm. 


\section{Further Algebraic Techniques}

\subsection{Localisation at a Single Index}

Let $\mathbb{V}$ be a zigzag module of length $n$ and let $1 \leq k \leq n$. We consider the problem of determining the set of intervals in Pers $(\mathbb{V})$ which contain $k$, without necessarily computing Pers $(\mathbb{V})$ itself. We shall see that all the necessary information is contained in a pair of filtrations on the vector space $V_{k}$.

Let $\mathbb{V}$ be a zigzag module of length $n$. The left-filtration of $\mathbb{V}$ is a filtration on $V_{1}$ of depth $n$, defined as

$$
\mathrm{L}(\mathbb{V})=\mathrm{R}(\overline{\mathbb{V}})
$$

where $\overline{\mathbb{V}}$ is the reversal of $\mathbb{V}$, with type $\bar{\tau}$; so $\bar{V}_{i}=V_{n+1-i}$, with maps $\bar{f}_{i}=g_{n-i}$ or $\bar{g}_{i}=f_{n-i}$.

For any $k$ we therefore have two natural filtrations on $V_{k}$ :

$$
\begin{aligned}
\mathcal{R}_{k} & =\left(R_{0}, R_{1}, \ldots, R_{k}\right)=\mathrm{R}(\mathbb{V}[1, k]), \\
\mathcal{L}_{k} & =\left(L_{0}, L_{1}, \ldots, L_{n+1-k}\right)=\mathrm{L}(\mathbb{V}[k, n]) ;
\end{aligned}
$$

the right-filtration over the index set $\{1, \ldots, k\}$ and the left-filtration over the index set $\{k, \ldots, n\}$. We also have birth-time and death-time indices

$$
\begin{aligned}
& \mathrm{b}_{k}=\left(b_{1}, \ldots, b_{k}\right)=\mathrm{b}(\tau[1, k]), \\
& \mathrm{d}_{k}=\left(d_{1}, \ldots, d_{n+1-k}\right)=n+1-\mathrm{b}(\bar{\tau}[k, n])
\end{aligned}
$$

which indicate the birth and death times associated with the respective subquotients of $\mathcal{R}_{k}$ and $\mathcal{L}_{k}$. These depend on the type $\tau$ of $\mathbb{V}$.

Example 5.1 Consider the zigzag module

$$
\mathbb{V}=\left(V_{1} \stackrel{f_{1}}{\longrightarrow} V_{2} \stackrel{f_{2}}{\longrightarrow} V_{3} \stackrel{g_{3}}{\longleftarrow} V_{4}\right) .
$$

At $k=2$, for instance, we have

$$
\begin{aligned}
\mathcal{R}_{2} & =\left(0, f_{1}\left(V_{1}\right), V_{2}\right), \\
\mathcal{L}_{2} & =\left(0, f_{2}^{-1}(0), f_{2}^{-1} g_{3}\left(V_{4}\right), V_{2}\right)
\end{aligned}
$$

and

$$
\begin{aligned}
& \mathrm{b}_{2}=(1,2), \\
& \mathrm{d}_{2}=(2,4,3) .
\end{aligned}
$$

We can now state the main theorem of this section.

Theorem 5.2 (Localisation at index $k$ ) Let $\mathbb{V}$ be a zigzag module of length $n$ and let $1 \leq k \leq n$. Let $\mathcal{R}_{k}, \mathcal{L}_{k}$ denote the right-and left-filtrations at $k$, and let $\mathrm{b}_{k}, \mathrm{~d}_{k}$ denote 
the birth-time and death-time indices at $k$. Then, for all $i, j$ in the range $1 \leq i \leq k$, $1 \leq j \leq n+1-k$, the multiplicity of $\left[b_{i}, d_{j}\right]$ in $\operatorname{Pers}(\mathbb{V})$ is equal to ${ }^{1}$

$$
\begin{aligned}
c_{i j}= & \operatorname{dim}\left(R_{i} \cap L_{j}\right)-\operatorname{dim}\left(R_{i-1} \cap L_{j}\right) \\
& -\operatorname{dim}\left(R_{i} \cap L_{j-1}\right)+\operatorname{dim}\left(R_{i-1} \cap L_{j-1}\right) .
\end{aligned}
$$

Remark Equivalently, $c_{i j}=\operatorname{dim}\left(\left(R_{i} \cap L_{j}\right) /\left(\left(R_{i-1} \cap L_{j}\right)+\left(R_{i} \cap L_{j-1}\right)\right)\right)$, the dimension of the $(i, j)$-th bifiltration subquotient.

This theorem answers the original question, because every interval containing $k$ can be written as $\left[b_{i}, d_{j}\right]$ for some choice of $i, j$. We now work towards a proof of Theorem 5.2.

Proposition 5.3 It is sufficient to prove Theorem 5.2 in the special case where $\mathbb{V}$ is right-streamlined over $\{1, \ldots, k\}$ and left-streamlined over $\{k, \ldots, n\}$.

Proof It is clear from Lemma 4.3 that we can write $\mathbb{V}=\mathbb{U} \oplus \mathbb{W}$ where $\mathbb{U}$ is supported in $\{1, \ldots, k-1\}$ and $\mathbb{W}$ is right-streamlined over $\{1, \ldots, k\}$. Indeed, take $\mathbb{U}=\mathbb{V}^{1} \oplus$ $\cdots \oplus \mathbb{V}^{k-1}$ and $\mathbb{W}=\mathbb{V}^{k} \oplus \cdots \oplus \mathbb{V}^{n}$. Moreover, it is sufficient to prove Theorem 5.2 for $\mathbb{W}$, because the filtrations $\mathcal{R}_{k}, \mathcal{L}_{k}$ remain unchanged from $\mathbb{V}$, and the discarded term $\mathbb{U}$ decomposes into intervals which do not contain $k$. Thus, we may assume that $\mathbb{V}$ is right-streamlined over $\{1, \ldots, k\}$.

Repeating this argument from the other side, we may further assume that $\mathbb{V}$ is left-streamlined over $\{k, \ldots, n\}$.

Proof of Theorem 5.2 Assume that $\mathbb{V}$ satisfies the condition in Proposition 5.3. It follows that every interval in Pers $(\mathbb{V})$ contains $k$ : any other interval in the decomposition would cause a failure of the streamline condition. We can therefore write the interval decomposition of $\mathbb{V}$ as

$$
\mathbb{V}=\bigoplus_{a \in A} \mathbb{I}_{a} \cong \bigoplus_{a \in A} \mathbb{I}\left(b_{p(a)}, d_{q(a)}\right)
$$

where $A$ indexes the summands, and $p: A \rightarrow\{1, \ldots, k\}$ and $q: A \rightarrow\{1, \ldots, n-$ $k+1\}$ identify the interval type of each summand in terms of the birth-time and death-time indices. It is apparent from this formulation that

$$
c_{i j}=\#\{a \in A \mid p(a)=i, q(a)=j\}
$$

and it remains to compute this in terms of the dimensions $\operatorname{dim}\left(R_{i} \cap L_{j}\right)$.

\footnotetext{
${ }^{1}$ The reader may notice a similarity between the formula for $c_{i j}$ and the definition of multiplicity in the persistence diagram given by Cohen-Steiner et al. in $[4,5]$. These are localisation formulas: what part of a two-parameter quantity, such as $\operatorname{dim}\left(R_{i} \cap L_{j}\right)$ or $\operatorname{rank}\left(V_{i} \rightarrow V_{j}\right)$, is contributed by a given rectangle in parameter space? 
The interval decomposition restricts at index $k$ to a direct-sum decomposition of $V_{k}$ into 1-dimensional subspaces $U_{a}$, generated by elements $x_{a}$, say. Then

$$
\mathcal{R}_{k}=\bigoplus_{a \in A} \mathrm{R}\left(\mathbb{I}_{a}[1, k]\right)=\bigoplus_{a \in A} \mathcal{R}_{k} \cap U_{a} \cong \bigoplus_{a \in A} \mathcal{J}(p(a), k),
$$

where the final isomorphism comes from Lemma 3.9. Now, the filtration subspace $R_{i}$ is spanned by the terms isomorphic to $\mathcal{J}(p, k)$ with $p \leq i$. In other words, for $i=$ $1, \ldots, k$ we have

$$
R_{i}=\operatorname{Span}\left\{x_{a} \mid p(a) \leq i\right\} .
$$

A similar argument proceeding from the other direction gives the analogous formula

$$
L_{j}=\operatorname{Span}\left\{x_{a} \mid q(a) \leq j\right\}
$$

for $j=1, \ldots, n+1-k$. Since the $x_{a}$ are independent, these formulas give bases for $R_{i}, L_{j}$.

We now claim that

$$
R_{i} \cap L_{j}=\operatorname{Span}\left\{x_{a} \mid p(a) \leq i, q(a) \leq j\right\}
$$

for all $i, j$. The inclusion Span $\leq R_{i} \cap L_{j}$ is obvious, because each of the spanning vectors $x_{a}$ belongs to both $R_{i}$ and $L_{j}$. In the other direction, if $x \in R_{i} \cap L_{j}$ then write $x=\sum_{a \in A} \lambda_{a} x_{a}$. Since $x \in R_{i}$, all the coefficients $\lambda_{a}$ with $p(a)>i$ must be zero. Since $x \in L_{j}$, all the coefficients $\lambda_{a}$ with $q(a)>j$ must be zero. Thus $x \in$ $\operatorname{Span}\left\{x_{a} \mid p(a) \leq i, q(a) \leq j\right\}$. This establishes the reverse inclusion $R_{i} \cap L_{j} \leq$ Span, and hence the equality.

Then

$$
\operatorname{dim}\left(R_{i} \cap L_{j}\right)=\#\left\{x_{a} \mid p(a) \leq i, q(a) \leq j\right\}=\sum_{p=1}^{i} \sum_{q=1}^{j} c_{p q}
$$

for all $i, j$. The formula in the theorem follows easily from this.

Remark The salient fact behind this result is that it is possible to find a direct-sum decomposition of $V_{k}$ which simultaneously decomposes the filtered spaces $\mathcal{R}_{k}, \mathcal{L}_{k}$ into intervals within their respective categories Filt $k$, Filt $t_{n+1-k}$. Here we achieved this by appealing to the interval decomposition of $\mathbb{V}$, but this can also be proved directly for an arbitrary pair of filtrations on a single vector space. The analogous statement for a triple of filtrations is false. For example

$$
\left(0, \mathrm{k} \oplus 0, \mathrm{k}^{2}\right), \quad\left(0,0 \oplus \mathrm{k}, \mathrm{k}^{2}\right), \quad\left(0, \Delta, \mathrm{k}^{2}\right),
$$

(where $\Delta=\{(x, x) \mid x \in \mathrm{k}\}$ ) cannot be simultaneously decomposed into intervals. 


\subsection{The Diamond Principle}

Consider the following diagram:

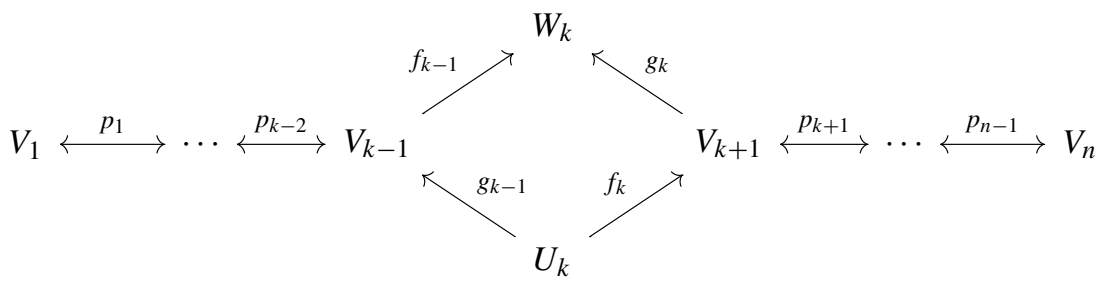

Let $\mathbb{V}^{+}$and $\mathbb{V}^{-}$denote the two zigzag modules contained in the diagram:

$$
\begin{aligned}
& \mathbb{V}^{+}=\left(V_{1} \longleftrightarrow \cdots \longleftrightarrow V_{k-1} \stackrel{f_{k-1}}{\longleftrightarrow} W_{k} \stackrel{g_{k}}{\longleftarrow} V_{k+1} \longleftrightarrow \cdots \longleftrightarrow V_{n}\right), \\
& \mathbb{V}^{-}=\left(V_{1} \longleftrightarrow \cdots \longleftrightarrow V_{k-1} \stackrel{g_{k-1}}{\longleftarrow} U_{k} \stackrel{f_{k}}{\longleftrightarrow} V_{k+1} \longleftrightarrow \cdots \longleftrightarrow V_{n}\right) .
\end{aligned}
$$

We wish to compare Pers $\left(\mathbb{V}^{+}\right)$with Pers $\left(\mathbb{V}^{-}\right)$, particularly with respect to intervals that meet $\{k-1, k, k+1\}$. This requires a favourable condition on the four maps in the middle diamond.

Definition 5.4 We say that the diagram

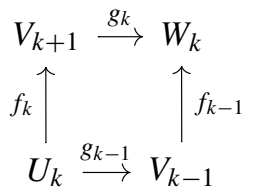

is exact if $\operatorname{Im}\left(D_{1}\right)=\operatorname{Ker}\left(D_{2}\right)$ in the following sequence

$$
U_{k} \stackrel{D_{1}}{\longrightarrow} V_{k-1} \oplus V_{k+1} \stackrel{D_{2}}{\longrightarrow} W_{k},
$$

where $D_{1}(u)=g_{k-1}(u) \oplus f_{k}(u)$ and $D_{2}\left(v \oplus v^{\prime}\right)=f_{k-1}(v)-g_{k}\left(v^{\prime}\right)$.

Theorem 5.5 (The Diamond Principle) Given $\mathbb{V}^{+}$and $\mathbb{V}^{-}$as above, suppose that the middle diamond is exact. Then there is a partial bijection of the multisets Pers $\left(\mathbb{V}^{+}\right)$ and $\operatorname{Pers}\left(\mathbb{V}^{-}\right)$, with intervals matched according to the following rules:

- Intervals of type $[k, k]$ are unmatched.

- Type $[b, k]$ is matched with type $[b, k-1]$ and vice versa, for $b \leq k-1$.

- Type $[k, d]$ is matched with type $[k+1, d]$ and vice versa, for $d \geq k+1$.

- Type $[b, d]$ is matched with type $[b, d]$, in all other cases.

It follows that the restrictions $\left.\operatorname{Pers}\left(\mathbb{V}^{+}\right)\right|_{K},\left.\operatorname{Pers}\left(\mathbb{V}^{-}\right)\right|_{K}$ to the set $K=\{1, \ldots, n\} \backslash\{k\}$ are equal. 

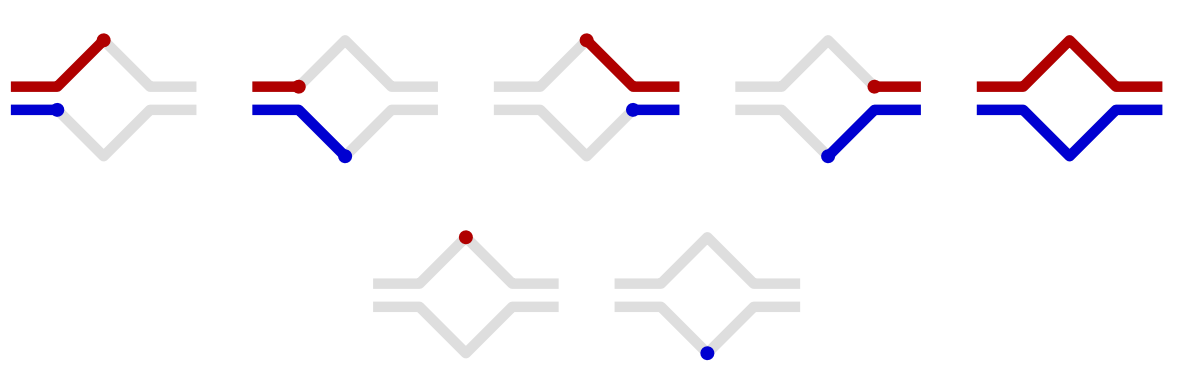

Fig. 5 Interval matching between Pers $\left(\mathbb{V}^{+}\right)$and Pers $\left(\mathbb{V}^{-}\right)$: (top row) the five cases where matching occurs; (bottom row) unmatched intervals $[k, k]$

Fig. 6 From $\operatorname{Pers}\left(\mathbb{V}^{+}\right)$to $\operatorname{Pers}\left(\mathbb{V}^{-}\right)$, for $n=6, k=4$ : points in the persistence plane move according to the arrows; the multiplicity of the point marked $\otimes$ changes unpredictably

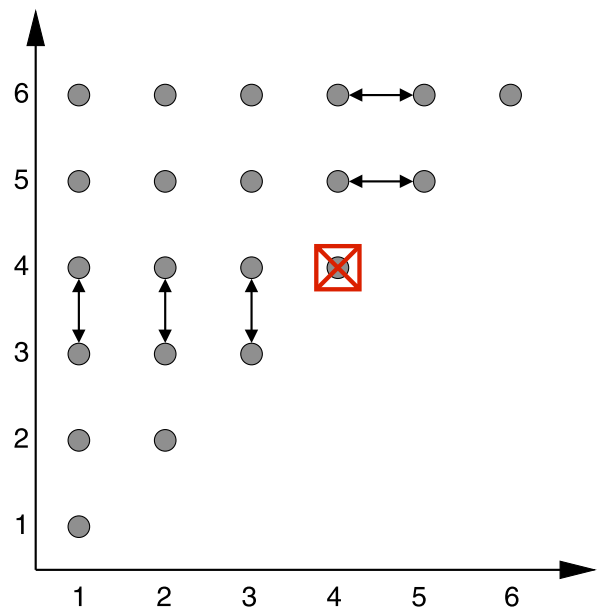

Figures 5 and 6 illustrate Theorem 5.5 in terms of barcodes and persistence diagrams, respectively.

Remark The $\mathbb{I}(k, k)$ summands in Pers $\left(\mathbb{V}^{+}\right)$span the cokernel of $D_{2}$, whereas the $\mathbb{I}(k, k)$ summands in Pers $\left(\mathbb{V}^{-}\right)$span the kernel of $D_{1}$. The hypothesis of Theorem 5.5 does not bring about any relation between these spaces (which is why the $[k, k]$ intervals are unmatched). In Sect. 5.3, however, we consider a situation in which the $[k, k]$ intervals can be tracked.

We use the localisation technique of Sect. 5.1 to prove Theorem 5.5. We begin with birth- and death-time indices.

Proposition 5.6 Let $\tau^{+}, \tau^{-}$denote the zigzag types of $\mathbb{V}^{+}, \mathbb{V}^{-}$respectively. If we write

$$
\left(b_{1}, \ldots, b_{k-1}\right)=\mathrm{b}\left(\tau^{+}[1, k-1]\right)=\mathrm{b}\left(\tau^{-}[1, k-1]\right)
$$


for the birth-time index up to time $k-1$, then

$$
\begin{aligned}
& \mathrm{b}\left(\tau^{+}[1, k+1]\right)=\left(k+1, b_{1}, \ldots, b_{k-1}, k\right), \\
& \mathrm{b}\left(\tau^{-}[1, k+1]\right)=\left(k, b_{1}, \ldots, b_{k-1}, k+1\right) .
\end{aligned}
$$

Similarly, if we write

$$
\left(d_{1}, \ldots, d_{n-k}\right)=\mathrm{d}\left(\tau^{+}[k+1, n]\right)=\mathrm{d}\left(\tau^{-}[k+1, n]\right)
$$

for the death-time index from time $k+1$, then

$$
\begin{aligned}
& \mathrm{d}\left(\tau^{+}[k-1, n]\right)=\left(k-1, d_{1}, \ldots, d_{n-k}, k\right), \\
& \mathrm{d}\left(\tau^{-}[k-1, n]\right)=\left(k, d_{1}, \ldots, d_{n-k}, k-1\right) .
\end{aligned}
$$

Proof This is immediate from the recursive definition of birth-time index. If we write $\tau_{0}=\tau^{+}[1, k-1]=\tau^{-}[1, k-1]$ then $\tau^{+}[1, k+1]=\tau_{0} f g$ and $\tau^{-}[1, k+1]=\tau_{0} g f$. The death-time index is treated similarly.

Here is the crux of the matter:

Lemma 5.7 In the situation of Theorem 5.5, the following filtrations are equal:

$$
\begin{aligned}
& \mathrm{R}\left(\mathbb{V}^{+}[1, k+1]\right)=\mathrm{R}\left(\mathbb{V}^{-}[1, k+1]\right), \\
& \mathrm{L}\left(\mathbb{V}^{+}[k-1, n]\right)=\mathrm{L}\left(\mathbb{V}^{-}[k-1, n]\right) .
\end{aligned}
$$

Proof Write $\left(R_{0}, R_{1}, \ldots, R_{k-1}\right)=\mathrm{R}\left(\mathbb{V}^{+}[1, k-1]\right)=\mathrm{R}\left(\mathbb{V}^{-}[1, k-1]\right)$. By the recursive formula (Definition 3.1),

$$
\mathrm{R}\left(\mathbb{V}^{+}[1, k+1]\right)=\left(0, g_{k}^{-1} f_{k-1}\left(R_{0}\right), \ldots, g_{k}^{-1} f_{k-1}\left(R_{k-1}\right), V_{k+1}\right)
$$

and

$$
\mathrm{R}\left(\mathbb{V}^{-}[1, k+1]\right)=\left(0, f_{k} g_{k-1}^{-1}\left(R_{0}\right), \ldots, f_{k} g_{k-1}^{-1}\left(R_{k-1}\right), V_{k+1}\right) .
$$

Thus we can prove the first statement of the lemma by showing that

$$
f_{k} g_{k-1}^{-1}(R)=g_{k}^{-1} f_{k-1}(R)
$$

for any subspace $R \leq V_{k-1}$. We use first-order logic. Let $x \in V_{k+1}$. We have the following chain of equivalent statements.

$$
\begin{aligned}
& x \in f_{k} g_{k-1}^{-1}(R) \\
\Leftrightarrow & (\exists z \in R)\left(\exists y \in U_{k}\right)\left(\left(g_{k-1}(y)=z\right) \&\left(f_{k}(y)=x\right)\right) \\
\Leftrightarrow & (\exists z \in R)\left(\exists y \in U_{k}\right)\left(D_{1}(y)=z \oplus x\right) \\
\Leftrightarrow & (\exists z \in R)\left(z \oplus x \in \operatorname{Im}\left(D_{1}\right)\right) .
\end{aligned}
$$


On the other hand:

$$
\begin{aligned}
& x \in g_{k}^{-1} f_{k-1}(R) \\
\Leftrightarrow & (\exists z \in R)\left(f_{k-1}(z)=g_{k}(x)\right) \\
\Leftrightarrow & (\exists z \in R)\left(z \oplus x \in \operatorname{Ker}\left(D_{2}\right)\right) .
\end{aligned}
$$

Since $\operatorname{Im}\left(D_{1}\right)=\operatorname{Ker}\left(D_{2}\right)$ by hypothesis, it follows that $f_{k} g_{k-1}^{-1}(R)=g_{k}^{-1} f_{k-1}(R)$.

This proves the first equality. The second equality follows symmetrically.

Proof of Theorem 5.5 We adopt the notation of Sect. 5.1, and consider the right- and left-filtrations at $V_{k+1}$, for both $\mathbb{V}^{+}$and $\mathbb{V}^{-}$. Since $\mathbb{V}^{+}[k+1, n]=\mathbb{V}^{-}[k+1, n]$ we have

$$
\mathcal{L}_{k+1}^{+}=\mathcal{L}_{k+1}^{-} \quad \text { and } \quad \mathrm{d}_{k+1}^{+}=\mathrm{d}_{k+1}^{-},
$$

and by Lemma 5.7 we have

$$
\mathcal{R}_{k+1}^{+}=\mathcal{R}_{k+1}^{-}
$$

Finally, $\mathrm{b}_{k+1}^{+}$agrees with $\mathrm{b}_{k+1}^{-}$except that $k, k+1$ are interchanged, according to Proposition 5.6. Thus, when we use Theorem 5.2 to calculate the multiplicity of $[b, d]$ for $b \leq k+1 \leq d$, there is perfect agreement between $\mathbb{V}^{+}$and $\mathbb{V}^{-}$except that we must interchange $k, k+1$ when they occur as birth times.

A symmetrical argument can be made, localising at $V_{k-1}$. When we compute the multiplicity of $[b, d]$ for $b \leq k-1 \leq d$, there is perfect agreement between $\mathbb{V}^{+}$and $\mathbb{V}^{-}$except that we must interchange $k, k-1$ when they occur as death times.

We have covered all cases of the theorem except for intervals which meet neither $k-1$ nor $k+1$. Intervals contained in [1,k-2] are automatically the same for $\mathbb{V}^{+}$and $\mathbb{V}^{-}$because they can be computed by restricting to $\mathbb{V}^{+}[1, k-1]$ and $\mathbb{V}^{-}[1, k-1]$, which are equal. Similarly, intervals contained in $[k+2, n]$ are the same for $\mathbb{V}^{+}$and $\mathbb{V}^{-}$, by restricting to $\mathbb{V}^{+}[k+1, n]=\mathbb{V}^{-}[k+1, n]$.

Finally, consider intervals $[k, k]$. Nothing can be said about those.

\subsection{The Strong Diamond Principle}

The Diamond Principle can usefully be applied to the following diagram of topological spaces and continuous maps. The four maps in the central diamond are inclusion maps, and the remaining maps $\leftrightarrow$ are arbitrary.

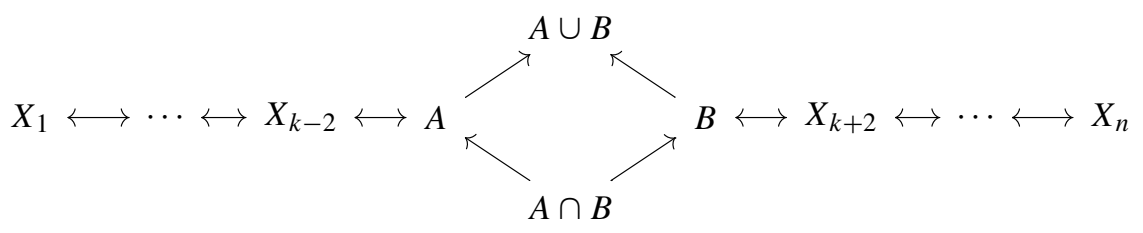

Let $\mathbb{X}^{+}, \mathbb{X}^{-}$denote the upper and lower zigzag diagrams contained in this picture; so $\mathbb{X}^{+}$passes through $A \cup B$, and $\mathbb{X}^{-}$passes through $A \cap B$. 
Theorem 5.8 (The Strong Diamond Principle) Given $\mathbb{X}^{+}$and $\mathbb{X}^{-}$as above, there is a (complete) bijection between the multisets $\operatorname{Pers}\left(\mathrm{H}_{*}\left(\mathbb{X}^{+}\right)\right)$and $\operatorname{Pers}\left(\mathrm{H}_{*}\left(\mathbb{X}^{-}\right)\right)$. Intervals are matched according to the following rules:

- $[k, k] \in \operatorname{Pers}\left(\mathrm{H}_{\ell+1}\left(\mathbb{X}^{+}\right)\right)$is matched with $[k, k] \in \operatorname{Pers}\left(\mathrm{H}_{\ell}\left(\mathbb{X}^{-}\right)\right)$.

In the remaining cases, the matching preserves homological dimension:

- Type $[b, k]$ is matched with type $[b, k-1]$ and vice versa, for $b \leq k-1$.

- Type $[k, d]$ is matched with type $[k+1, d]$ and vice versa, for $d \geq k+1$.

- Type $[b, d]$ is matched with type $[b, d]$, in all other cases.

Proof For any $\ell$, apply the homology functor $\mathrm{H}_{\ell}$ to the diagram. The central diamond

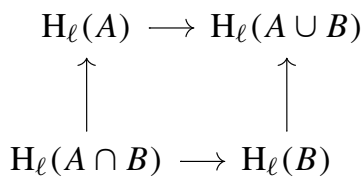

is exact by virtue of the Mayer-Vietoris theorem, according to which

$$
\cdots \longrightarrow \mathrm{H}_{\ell}(A \cap B) \stackrel{D_{1}}{\longrightarrow} \mathrm{H}_{\ell}(A) \oplus \mathrm{H}_{\ell}(B) \stackrel{D_{2}}{\longrightarrow} \mathrm{H}_{\ell}(A \cup B) \longrightarrow \cdots
$$

is an exact sequence. The Diamond Principle therefore applies to $\mathrm{H}_{\ell}\left(\mathbb{X}^{+}\right)$and $\mathrm{H}_{\ell}\left(\mathbb{X}^{-}\right)$, and we have a partial bijection which accounts for all intervals except those of type $[k, k]$.

Now consider the connecting homomorphism in the same Mayer-Vietoris sequence:

$$
\cdots \stackrel{D_{2}}{\longrightarrow} \mathrm{H}_{\ell+1}(A \cup B) \stackrel{\partial}{\longrightarrow} \mathrm{H}_{\ell}(A \cap B) \stackrel{D_{1}}{\longrightarrow} \cdots .
$$

By exactness, $\partial$ induces an isomorphism between the cokernel of $D_{2}$ and the kernel of $D_{1}$. But the $[k, k]$ summands of $\operatorname{Pers}\left(\mathrm{H}_{\ell+1}\left(\mathbb{X}^{+}\right)\right)$precisely span $\operatorname{Coker}\left(D_{2}\right)$, whereas the $[k, k]$ summands of $\operatorname{Pers}\left(\mathrm{H}_{\ell}\left(\mathbb{X}^{-}\right)\right)$span $\operatorname{Ker}\left(D_{1}\right)$. This establishes the claimed bijection between the $[k, k]$ intervals.

Example 5.9 Let $\mathbb{X}=\left(X_{1}, \ldots, X_{n}\right)$ be a sequence of simplicial complexes defined on a common vertex set. Suppose these have arisen in some context where each transition $X_{i}$ to $X_{i+1}$ is regarded as being a 'small' change. There are two natural zigzag sequences linking the $X_{i}$.

The union zigzag, $\mathbb{X}_{\cup}$ :

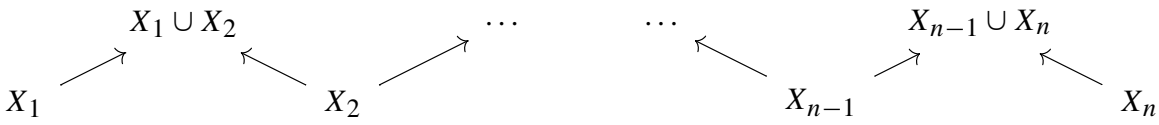


Fig. 7 From Pers $\left(\mathrm{H}_{*}\left(\mathbb{X}_{\cap}\right)\right)$ to $\operatorname{Pers}\left(\mathrm{H}_{*}(\mathbb{X} \cup)\right)$, for $n=5$ : points in the persistence plane move according to the arrows; points marked $\oplus$ stay fixed and increase homological dimension by 1

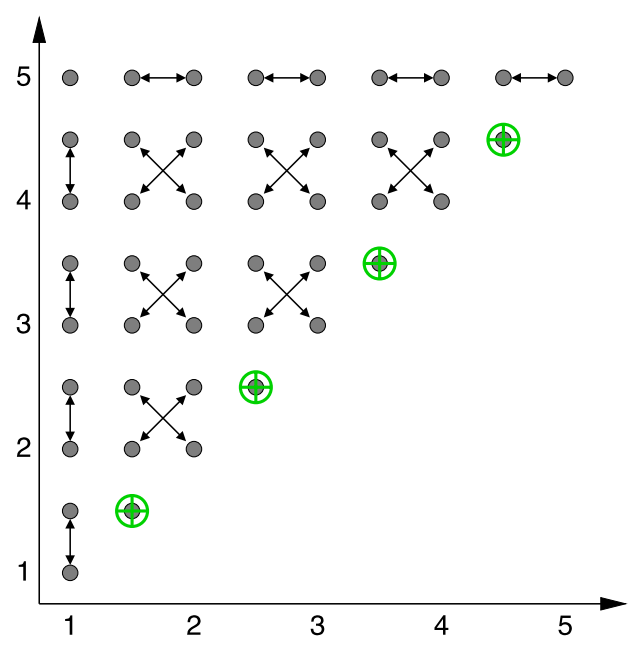

The intersection zigzag, $\mathbb{X}_{\cap}$ :
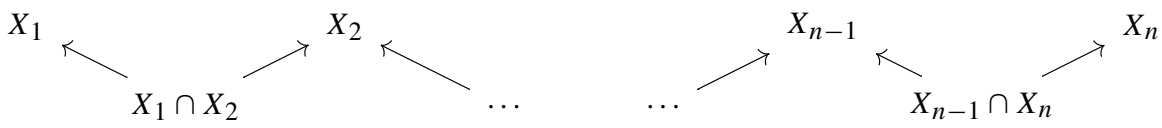

We can think of these as being indexed by the half-integers $\left\{1,1 \frac{1}{2}, 2,2 \frac{1}{2}, \ldots, n\right\}$.

We can apply the Strong Diamond Principle $n-1$ times to derive the following relationship between the zigzag persistence of the two sequences $\operatorname{Pers}\left(\mathrm{H}_{\ell}\left(\mathbb{X}_{\cap}\right)\right)$ and $\operatorname{Pers}\left(\mathrm{H}_{\ell}\left(\mathbb{X}_{\cup}\right)\right)$. Restricting to the integer indices, there is a coarse equality:

$$
\left.\operatorname{Pers}\left(\mathrm{H}_{\ell}\left(\mathbb{X}_{\cup}\right)\right)\right|_{\{1, \ldots, n\}}=\left.\operatorname{Pers}\left(\mathrm{H}_{\ell}\left(\mathbb{X}_{\cap}\right)\right)\right|_{\{1, \ldots, n\}} .
$$

More finely, there is a partial bijection between $\operatorname{Pers}\left(\mathrm{H}_{\ell}\left(\mathbb{X}_{\cup}\right)\right)$ and $\operatorname{Pers}\left(\mathrm{H}_{\ell}\left(\mathbb{X}_{\cap}\right)\right)$. Intervals $\left[k \frac{1}{2}, k \frac{1}{2}\right]$ shift homological dimension by +1 (from the intersection sequence to the union sequence). Otherwise $[b, d] \leftrightarrow\left[b^{\prime}, d^{\prime}\right]$ where $\left\{b, b^{\prime}\right\}$ is an unordered pair of the form $\left\{k \frac{1}{2}, k+1\right\}$ and $\left\{d, d^{\prime}\right\}$ is an unordered pair of the form $\left\{k, k \frac{1}{2}\right\}$; dimension is preserved. Figure 7 illustrates the complete correspondence as a transformation of the persistence diagram, for $n=5$.

The Pyramid Theorem of [3] is a powerful extension of these ideas.

\section{Concluding Remarks}

We have presented the foundations of a theory of zigzag persistence which, we believe, considerably extends and enriches the well known and highly successful theory of persistent homology. Zigzag persistence originates in the work of Gabriel and others in the theory of quiver representations. One of our goals has been to bridge the 
gap between the quiver literature (which is read largely by algebraists) and the current language of applied and computational topology. To this end, we have described an algorithmic form of Gabriel's structure theorem for $A_{n}$ quivers, and have indicated the first steps towards integrating these ideas into tools for applied topology.

There are several ways in which this work is incomplete. The most significant omission is an algorithm for computing zigzag persistence in a homological setting (as distinct from the somewhat sanitised vector space algorithm described in Sect. 4.3). We address this gap in a paper with Dmitriy Morozov [3], where we present an algorithm for computing the zigzag persistence intervals of a 1-parameter family of simplicial complexes on a fixed vertex set.

We have made no effort in this paper to flesh out the applications suggested in Sect. 1. There is often a substantial gap between the concrete world of point-cloud data sets and the ideal world of simplicial complexes and topological spaces. We intend to develop some of these applications in future work.

Meanwhile, we have given priority to establishing the theoretical language and tools. The Diamond Principle is particularly powerful. In the paper with Morozov [3], we use the Diamond Principle to establish isomorphisms between several different classes of persistence invariants of a space with a real-valued (e.g. Morse) function. In particular, we use zigzag persistence to resolve an open conjecture concerning extended persistence [6]. This supports our contention that zigzag persistence provides the appropriate level of generality and power for understanding the heuristic concept of persistence in its many manifestations.

\section{Index of Notation}

\begin{tabular}{lll}
\hline Symbol & Description & Section \\
\hline $\mathrm{k}$ & coefficient field (fixed throughout) & 2 \\
$\tau$ & zigzag module type (e.g. $f f g$ ) & 2.1 \\
$\tau$ Mod & category of zigzag modules of type $\tau$ & 2.1 \\
$\mathbb{V}$ & zigzag module & 2.1 \\
$V_{i}$ & vector space of $\mathbb{V}$ at index $i$ & 2.1 \\
Pers $(\mathbb{V})$ & zigzag persistence of $\mathbb{V}$ & 2.3 \\
{$[b, d]$} & closed interval $\{b, \ldots, d\} \subseteq\{1, \ldots, n\}$ & 2.2 \\
$\mathbb{I}_{\tau}(b, d)$ & interval zigzag module (type $\tau$ may be omitted) & 2.2 (Def. 2.3) \\
$\mathrm{R}(\mathbb{V})$ & right-filtration of $\mathbb{V}$ & 3.1 (Def. 3.1) \\
$\mathrm{b}(\tau)$ & birth-time index vector for $\tau$ & 3.1 (Def. 3.6) \\
$\mathrm{L}(\mathbb{V})$ & left-filtration of $\mathbb{V}$ & 5.1 \\
$\mathrm{~d}(\tau)$ & death-time index vector for $\tau$ & 5.1 \\
Filt $n$ & category of filtered vector spaces of depth $n$ & 3.2 \\
$\mathcal{R}$ & filtered vector space & 3.2 \\
$R_{i}$ & vector space at level $i$ of $\mathcal{R}$ & 3.2 \\
$\mathcal{R} \cap K$ & & \\
$\operatorname{dim}(\mathcal{R})$ & induced subspace of $\mathcal{R}$ & 3.2 \\
$\mathcal{J}(i, n)$ & dimension vector of $\mathcal{R}$ & 3.2 \\
& interval filtered vector space & 3.1 (Lem. 3.9)
\end{tabular}




\begin{tabular}{lll}
\hline Symbol & Description & Section \\
\hline & & \\
$\mathbb{V}[p, q]$ & restriction of $\mathbb{V}$ to index range $\{p, \ldots, q\}$ & 2.3 (Def. 2.7) \\
$\mathbb{V}[k]$ & truncation of $\mathbb{V}$ to index range $\{1, \ldots, k\}$ & 4.1 \\
$\left.\operatorname{Pers}(\mathbb{V})\right|_{K}$ & restriction of Pers $(\mathbb{V})$ to index set $K \subset\{1, \ldots, n\}$ & 2.3 (Rem. 2.13) \\
\hline
\end{tabular}

Acknowledgements The authors thank Greg Kuperberg, Konstantin Mischaikow and Dmitriy Morozov for helpful conversations; Mikhail Khovanov for valuable correspondence; Jennifer Novak Kloke, Peter Landweber and the anonymous referees for detailed feedback on our manuscript. The authors gratefully acknowledge support from DARPA, in the form of grants HR0011-05-1-0007 (TDA) and HR0011-07-10002 (SToMP). The second author wishes to thank Pomona College and Stanford University for, respectively, granting and hosting his sabbatical during late 2008. The second author holds a Digiteo Chair.

Open Access This article is distributed under the terms of the Creative Commons Attribution Noncommercial License which permits any noncommercial use, distribution, and reproduction in any medium, provided the original author(s) and source are credited.

\section{References}

1. M.F. Atiyah, On the Krull-Schmidt theorem with application to sheaves, Bull. Soc. Math. France 84, 307-317 (1956).

2. G. Carlsson, T. Ishkhanov, V. de Silva, A. Zomorodian, On the local behavior of spaces of natural images, Int. J. Comput. Vis. 76(1), 1-12 (2008).

3. G. Carlsson, V. de Silva, D. Morozov, Zigzag persistent homology and real-valued functions, in Proceedings 25th ACM Symposium on Computational Geometry (SoCG), 2009, pp. 247-256.

4. F. Chazal, D. Cohen-Steiner, M. Glisse, L. Guibas, S. Oudot, Proximity of persistence modules and their diagrams, in Proceedings of the 25th Annual ACM Symposium on Computational Geometry (SoCG), 2009, pp. 237-246.

5. D. Cohen-Steiner, H. Edelsbrunner, J. Harer, Stability of persistence diagrams, Discrete Comput. Geom. 37(1), 103-120 (2007).

6. D. Cohen-Steiner, H. Edelsbrunner, J. Harer, Extending persistence using Poincaré and Lefschetz duality. Found. Comput. Math. 9(1), 79-103 (2009).

7. V. de Silva, G. Carlsson, Topological estimation using witness complexes, in Eurographics Symposium on Point-Based Graphics, ed. by M. Alexa, S. Rusinkiewicz (ETH, Zürich, 2004), pp. 157-166.

8. H. Derksen, J. Weyman, Quiver representations, Not. Am. Math. Soc. 52(2), 200-206 (2005).

9. H. Edelsbrunner, E.P. Mücke, Three-dimensional alpha shapes, ACM Trans. Graph. 13(1), 43-72 (1994).

10. H. Edelsbrunner, D. Letscher, A. Zomorodian, Topological persistence and simplification, Discrete Comput. Geom. 28, 511-533 (2002).

11. P. Gabriel, Unzerlegbare Darstellungen I, Manuscr. Math. 6, 71-103 (1972).

12. V.G. Kac, Infinite root systems, representations of graphs and invariant theory, Invent. Math. 56(1), 57-92 (1980).

13. S. Lang, Algebra, 3rd edn. Graduate Texts in Mathematics (Springer, Berlin, 2005).

14. A. Zomorodian, G. Carlsson, Computing persistent homology, Discrete Comput. Geom. 33(2), 249274 (2005). 